Supporting information for the manuscript entitled

\title{
Total Synthesis of (-)-Bitungolide F and Determination of Its Absolute Stereochemistry
}

\author{
Subhash Ghosh, Soma Uday Kumar and J. Shashidhar \\ Indian Institute of Chemical Technology, Hyderabad 500 007, India \\ subhash@iict.res.in
}

\section{Table of Contents}

1. Experimental procedures and data for the compounds

$17-20,12,26,11,28-29,10,30-32,9$ and 34

2. Spectra $\left({ }^{1} \mathrm{H}\right.$ and $\left.{ }^{13} \mathrm{C} \mathrm{NMR}\right)$ of compounds

S 16- S 36

$17-20,12,25-26,11,27-29,10,30-32,9,33-35,6$, and

natural bitungolide $\mathrm{F}$. 
General Experimental Procedures. All reactions were carried out in oven or flamedried glassware with magnetic stirring under nitrogen atmosphere using dry, freshly distilled solvents, unless otherwise noted. Reactions were monitored by thin layer chromatography (TLC) carried out on $0.25 \mathrm{~mm}$ silica gel plates with UV light, $\mathrm{I}_{2}, 7 \%$ ethanolic phosphomolybdic acid-heat and 2.5\% ethanolic anisaldehyde (with $1 \% \mathrm{AcOH}$ and $3.3 \%$ conc. $\mathrm{H}_{2} \mathrm{SO}_{4}$ )-heat as developing agents. Silica gel finer than 200 mesh was used for flash column chromatography. Yields refer to chromatographically and spectroscopically homogeneous materials unless otherwise stated. Melting points are uncorrected. IR spectra were recorded as neat liquids or $\mathrm{KBr}$ pellets. Mass spectra were obtained under electron impact ionisation (EI), liquid secondary ion mass spectrometric (LSIMS) technique, electron spray ionisation (ESI) and MALDI techniques. Optical rotations were measured with a digital polarimeter. NMR spectra were recorded on 500, 300 and $200 \mathrm{MHz}$ spectrometers at $30^{\circ} \mathrm{C}$ with $2-10 \mathrm{mM}$ solutions in appropriate solvents using TMS as internal standard or the solvent signals as secondary standards and the chemical shifts are shown in $\delta$ scales. Multiplicities of NMR signals are designated as s (singlet), d (doublet), t (triplet), q (quartet), br (broad), m (multiplet, for unresolved lines), etc. ${ }^{13} \mathrm{C}$ NMR spectra were recorded on $150,100,75$ and $50 \mathrm{MHz}$ spectrometers with complete proton decoupling. 
(4S)-4-Benzyl-3-((2S,3R,4R)-5-[1-(tert-butyl)-1,1-diphenylsilyl]oxy-2-ethyl- 3hydroxy-4-methylpentanoyl)-1,3-oxazolan-2-one (17):

To the compound $16(5.07 \mathrm{~g}, 20.3 \mathrm{mmol})$ in dry $\mathrm{CH}_{2} \mathrm{Cl}_{2}(50 \mathrm{~mL})$ at $0{ }^{\circ} \mathrm{C}$ was added $\mathrm{TiCl}_{4}(2.3 \mathrm{~mL}, 21.2 \mathrm{mmol})$ and after $5 \mathrm{~min}$, (-)-sparteine $(11.1 \mathrm{~mL}, 48.19 \mathrm{mmol})$ was added. After stirring at the same temperature for $20 \mathrm{~min}$, aldehyde $\mathbf{1 4}(6.1 \mathrm{~g}, 18.6$ mmol) was added as a solution of dry $\mathrm{CH}_{2} \mathrm{Cl}_{2}$ under nitrogen atmosphere. The reaction mixture was quenched after 10 min with half saturated aqueous $\mathrm{NH}_{4} \mathrm{Cl}$ solution $(30 \mathrm{~mL})$ and extracted with EtOAc $(2 \times 300 \mathrm{~mL})$. The combined organic layers were washed with water $(100 \mathrm{~mL}), 0.5 \mathrm{~N} \mathrm{HCl}(30 \mathrm{~mL})$, brine $(50 \mathrm{~mL})$, dried $\left(\mathrm{Na}_{2} \mathrm{SO}_{4}\right)$ and concentrated in vacuo. Purification of the crude residue by column chromatography (18-22\% EtOAc in petroleum ether) provided compound $\mathbf{1 7}$ (11.3 g, 95\% yield) as a white solid. Optical rotation $[\alpha]_{\mathrm{D}}^{22}=+7.1\left(\right.$ c $\left.1, \mathrm{CHCl}_{3}\right)$; IR (neat): $v_{\max } 3492(\mathrm{br}), 2961,1781,1694,1386$, 1208, 820, 746 and $702 \mathrm{~cm}^{-1}$; ${ }^{1} \mathrm{H}$ NMR $\left(\mathrm{CDCl}_{3}, 300 \mathrm{MHz}\right): \delta 7.69-7.65(\mathrm{~m}, 4 \mathrm{H}), 7.43-$ $7.37(\mathrm{~m}, 6 \mathrm{H}), 7.34-7.21(\mathrm{~m}, 5 \mathrm{H}) 4.76-4.67(\mathrm{~m}, 1 \mathrm{H}), 4.15(\mathrm{~d}, J=4.9 \mathrm{~Hz}, 2 \mathrm{H}), 4.01(\mathrm{td}, J=$ 3.7, $9.8 \mathrm{~Hz}, 1 \mathrm{H}), 3.91$ (dd, $J=3.7,7.5 \mathrm{~Hz}, 1 \mathrm{H}), 3.82$ (dd, $J=4.1,10.2 \mathrm{~Hz}, 1 \mathrm{H}), 3.77$ (brs, 1H), $3.68(\mathrm{dd}, J=6.4,10.2 \mathrm{~Hz}, 1 \mathrm{H}), 3.37(\mathrm{dd}, J=3.0,13.2 \mathrm{~Hz}, 1 \mathrm{H}), 2.74(\mathrm{dd}, J=9.8$, 13.2 Hz, 1H), 2.15-2.02 (m, 1H), 1.92-1.83 (m, 1H), 1.78-1.65 (m, 1H), $1.05(\mathrm{~s}, 9 \mathrm{H}), 1.01$ (t, $J=7.5 \mathrm{~Hz}, 3 \mathrm{H})$ and $0.95(\mathrm{~d}, J=7.1 \mathrm{~Hz}, 3 \mathrm{H}) ;{ }^{13} \mathrm{C} \mathrm{NMR}\left(\mathrm{CDCl}_{3}, 75 \mathrm{MHz}\right): \delta 175.3$, 153.1, 135.6, 135.5, 132.9, 129.8, 129.3, 128.9, 127.7, 127.3, 75.7, 68.5, 65.9, 55.7, 47.6, 37.9, 26.8, 26.7, 19.1, 18.4, 13.6 and 11.9; HRMS (ESIMS): calcd for $\mathrm{C}_{34} \mathrm{H}_{43} \mathrm{NO}_{5} \mathrm{NaSi}$ $[\mathrm{M}+\mathrm{Na}]^{+}: 596.2802$, found: 596.2834 .

$(2 R, 3 R, 4 R)-5$-[1-(tert-Butyl)-1,1-diphenylsilyl]oxy-2-ethyl-4-methylpentane-1,3-diol (18): 
To a solution of compound $\mathbf{1 7}(11.3 \mathrm{~g}, 19.8 \mathrm{mmol})$ in dry ether at $0{ }^{\circ} \mathrm{C}$ were added one drop of distilled water followed by $\mathrm{LiBH}_{4}(860 \mathrm{mg}, 39.6 \mathrm{mmol})$ in portion wise and stirred at the same temperature for $10 \mathrm{~min}$. The reaction was then quenched with careful addition of distilled water and extracted with EtOAc $(2 \times 100 \mathrm{~mL})$, the combined organic layers were washed with water $(50 \mathrm{~mL})$, brine $(50 \mathrm{~mL})$, dried $\left(\mathrm{Na}_{2} \mathrm{SO}_{4}\right)$ and concentrated in vacuo. Purification of the crude residue by column chromatography $(25 \%$ EtOAc in petroleum ether) provided compound $\mathbf{1 8}(6.67 \mathrm{~g}, 84 \%$ yield) as white solid. Optical rotation $[\alpha]_{\mathrm{D}}^{22}=-21.51\left(\right.$ c $\left.3.1, \mathrm{CHCl}_{3}\right)$; IR (KBr): $v_{\max } 3427(\mathrm{br}), 2959,2864,1465$, 1109, 741 and $702 \mathrm{~cm}^{-1} ;{ }^{1} \mathrm{H}$ NMR $\left(\mathrm{CDCl}_{3}, 200 \mathrm{MHz}\right): \delta 7.68-7.63(\mathrm{~m}, 4 \mathrm{H}), 7.45-7.35(\mathrm{~m}$, $6 \mathrm{H}), 4.38-4.36(\mathrm{~m}, 1 \mathrm{H}), 3.90-3.80(\mathrm{~m}, 3 \mathrm{H}), 3.73(\mathrm{dd}, J=4.4,10.2 \mathrm{~Hz}, 1 \mathrm{H}), 3.0$ (brs, $1 \mathrm{H})$, 2.02-1.89 (m, 1H), 1.79-1.62 (m, 1H), 1.46-1.34 (m, 1H), $1.06(\mathrm{~s}, 9 \mathrm{H}), 0.99(\mathrm{t}, J=7.3$ $\mathrm{Hz}, 3 \mathrm{H})$ and $0.66(\mathrm{~d}, J=7.3 \mathrm{~Hz}, 3 \mathrm{H}) ;{ }^{13} \mathrm{C} \mathrm{NMR}\left(\mathrm{CDCl}_{3}, 50 \mathrm{MHz}\right): \delta 135.5,132.2,129.9$, $127.8,81.4,70.4,64.7,42.9,37.1,26.7,18.9,15.4,12.8$ and 12.1; HRMS (ESIMS): calcd for $\mathrm{C}_{24} \mathrm{H}_{36} \mathrm{O}_{3} \mathrm{NaSi}[\mathrm{M}+\mathrm{Na}]^{+}: 423.2331$, found: 423.2352 .

\section{tert-Butyl((2R)-2-[(4R,5R)-5-ethyl-2-(4-methoxyphenyl)-1,3-dioxan-4- yl]propyloxy)diphenylsilane (19):}

To the compound $18(6.28 \mathrm{~g}, 15.7 \mathrm{mmol})$ in dry $\mathrm{CH}_{2} \mathrm{Cl}_{2}(35 \mathrm{~mL})$ at $0{ }^{\circ} \mathrm{C}$ was added freshly prepared PMP acetal $(4.1 \mathrm{~mL}, 6.35 \mathrm{mmol})$, CSA (360 mg, $1.57 \mathrm{mmol})$ and stirred the resulting reaction mixture at the ambient temperature for $6 \mathrm{~h}$. The reaction mixture was quenched with saturated aqueous $\mathrm{NaHCO}_{3}$ solution $(60 \mathrm{~mL})$ and extracted with EtOAc $(2 \times 175 \mathrm{~mL})$. Combined organic layers were washed with water $(90 \mathrm{~mL})$, brine $(90 \mathrm{~mL})$, dried $\left(\mathrm{Na}_{2} \mathrm{SO}_{4}\right)$ and concentrated in vacuo. Purification of the crude residue by column chromatography (10-12\% EtOAc in petroleum ether) provided 
compound $19(6.4 \mathrm{~g}, 94 \%$ yield $)$ as colorless oil. Optical rotation $[\alpha]_{\mathrm{D}}{ }^{22}=+7.88$ (c 2.45, $\mathrm{CHCl}_{3}$ ); IR (neat): $v_{\max } 2960,2857,1463,1248,1110,824$ and $703 \mathrm{~cm}^{-1} ;{ }^{1} \mathrm{H}$ NMR $\left(\mathrm{CDCl}_{3}, 300 \mathrm{MHz}\right): \delta$ 7.68-7.59; (m, 4H, Ar- $\left.H\right), 7.39-7.30(\mathrm{~m}, 6 \mathrm{H}), 7.19(\mathrm{~d}, J=7.5 \mathrm{~Hz}$, 2H), $6.85(\mathrm{~d}, J=9.1 \mathrm{~Hz}, 2 \mathrm{H}), 5.44(\mathrm{~s}, 1 \mathrm{H}), 4.3(\mathrm{~d}, J=11.3 \mathrm{~Hz}, 1 \mathrm{H}), 3.97-3.90(\mathrm{~m}, 3 \mathrm{H})$, $3.80(\mathrm{~s}, 3 \mathrm{H}), 3.65(\mathrm{dd}, J=2.3,9.8 \mathrm{~Hz}, 1 \mathrm{H}), 1.97-1.73(\mathrm{~m}, 2 \mathrm{H}), 1.55-1.42(\mathrm{~m}, 1 \mathrm{H}), 1.37-$ $1.28(\mathrm{~m}, 1 \mathrm{H}), 1.05(\mathrm{~s}, 9 \mathrm{H}), 1.03(\mathrm{~d}, J=6.8 \mathrm{~Hz}, 3 \mathrm{H})$ and $1.0(\mathrm{t}, J=7.5 \mathrm{~Hz}, 3 \mathrm{H}) ;{ }^{13} \mathrm{C} \mathrm{NMR}$ $\left(\mathrm{CDCl}_{3}, 75 \mathrm{MHz}\right): \delta 159.7,135.6,135.5,133.9,131.7,129.5,127.6,127.3,113.5,101.6$ 80.3, 69.5, 64.7, 55.3, 36.6, 26.9, 19.4, 16.3, 12.5 and 12.0; HRMS (ESIMS): calcd for $\mathrm{C}_{32} \mathrm{H}_{42} \mathrm{O}_{4} \mathrm{NaSi}[\mathrm{M}+\mathrm{Na}]^{+}: 541.2750$, found: 541.2771.

\section{(2R)-2-[(4R,5R)-5-Ethyl-2-(4-methoxyphenyl)-1,3-dioxan-4-yl]propan-1-ol (20):}

To a stirred solution of compound $19(6.38 \mathrm{~g}, 11.6 \mathrm{mmol})$ in dry THF $(25 \mathrm{~mL})$, TBAF (1M solution in THF $12.0 \mathrm{~mL}, 11.6 \mathrm{mmol})$ was added at $0{ }^{\circ} \mathrm{C}$. Reaction mixture was warmed to room temperature and stirred for $4 \mathrm{~h}$. It was quenched with saturated aqueous $\mathrm{NH}_{4} \mathrm{Cl}$ solution $(25 \mathrm{~mL})$, extracted with EtOAc $(2 \times 100 \mathrm{~mL})$, washed with brine (40 mL), dried $\left(\mathrm{Na}_{2} \mathrm{SO}_{4}\right)$ and concentrated in vacuo. Purification by column chromatography $\left(\mathrm{SiO}_{2}, 25 \%\right.$ EtOAc in petroleum ether) afforded pure compound 20 $(3.26 \mathrm{~g}, 92 \%)$ as colorless oil. Optical rotation $[\alpha]_{\mathrm{D}}^{22}=+17.25\left(c 0.8, \mathrm{CHCl}_{3}\right)$; IR (neat): $v_{\max }$ 3403(br), 2917, 2850, 1246, 1093, and $1034 \mathrm{~cm}^{-1} ;{ }^{1} \mathrm{H}$ NMR $\left(\mathrm{CDCl}_{3}, 200 \mathrm{MHz}\right): \delta$ 7.47-7.40 (m, 2H), 6.97-6.90 (m, 2H), $5.56(\mathrm{~s}, 1 \mathrm{H}), 4.35(\mathrm{dd}, J=1.4,11.5 \mathrm{~Hz}, 1 \mathrm{H}), 4.01-$ 3.89 (m, 2H), 3.85 (s, 3H), $3.76(\mathrm{dd}, J=7.2,10.8 \mathrm{~Hz}, 1 \mathrm{H}), 3.65(\mathrm{dd}, J=2.2,9.4 \mathrm{~Hz}, 1 \mathrm{H})$, $2.81($ brs, $1 \mathrm{H}), 2.21-2.04(\mathrm{~m}, 1 \mathrm{H}), 2.01-1.81(\mathrm{~m}, 1 \mathrm{H}), 1.72-1.50(\mathrm{~m}, 1 \mathrm{H}), 1.44-1.33(\mathrm{~m}$, $1 \mathrm{H}), 1.08(\mathrm{t}, J=7.2 \mathrm{~Hz}, 3 \mathrm{H})$ and $0.88(\mathrm{~d}, J=6.5 \mathrm{~Hz}, 3 \mathrm{H}) ;{ }^{13} \mathrm{C} \mathrm{NMR}\left(\mathrm{CDCl}_{3}, 75 \mathrm{MHz}\right): \delta$ 
$16.0,131.0,127.2,113.7,101.9,85.9,69.4,68.1,55.3,36.8,36.2,16.5,12.0$ and 11.9;

HRMS (ESIMS): calcd for $\mathrm{C}_{16} \mathrm{H}_{24} \mathrm{O}_{4} \mathrm{Na}[\mathrm{M}+\mathrm{Na}]^{+}: 303.1572$, found: 303.1560 .

\section{Dimethyl-(4S)-5-[1-(tert-butyl)-1,1-diphenylsilyl]oxy-2-oxo-4-[(1,1,1}

triethylsilyl)oxy]pentylphosphonate (12):

To the freshly distilled dimethyl methylphosphonate $(12.3 \mathrm{~mL}, 115.3 \mathrm{mmol})$ in dry THF was added ${ }^{n} \mathrm{BuLi}(1.6 \mathrm{M}$ solution in $n$-Hexane $70 \mathrm{~mL}, 111.9 \mathrm{mmol})$ drop wise at $-78{ }^{\circ} \mathrm{C}$ over $15 \mathrm{~min}$ and stirred at the same temperature for $1 \mathrm{~h}$. compound $24(13.79 \mathrm{~g}$, $28.8 \mathrm{mmol}$ ) was introduced into the reaction mixture slowly over a period of $10 \mathrm{~min}$ under nitrogen atmosphere as a solution of THF $(50 \mathrm{~mL})$. The resulting mixture was stirred for $1 \mathrm{~h}$ at the same temperature and then warmed to ambient temperature before quenching with saturated aqueous $\mathrm{NH}_{4} \mathrm{Cl}$ solution $(50 \mathrm{~mL})$ at $0{ }^{\circ} \mathrm{C}$. The product was extracted with EtOAc $(2 \times 250 \mathrm{~mL})$, washed with water $(100 \mathrm{~mL})$, brine $(100 \mathrm{~mL})$, dried $\left(\mathrm{Na}_{2} \mathrm{SO}_{4}\right)$ and concentrated in vacuo. Purification by column chromatography $\left(\mathrm{SiO}_{2}, 50-\right.$ 60\% EtOAc in petroleum ether) afforded pure compound $12(13.2 \mathrm{~g}, 81 \%)$ as colorless oil. Optical rotation $[\alpha]_{\mathrm{D}}^{22}=-19.59\left(\right.$ c $\left.2.2, \mathrm{CHCl}_{3}\right)$; IR (neat): $v_{\max } 2954,2877,2360$, 1717, 1260, 1110, 1033, 740 and $704 \mathrm{~cm}^{-1} ;{ }^{1} \mathrm{H}$ NMR $\left(\mathrm{CDCl}_{3}, 300 \mathrm{MHz}\right): \delta 7.68-7.63(\mathrm{~m}$, 4H), 7.43-7.34 (m, 6H), 4.20 (tt, $J=4.5,7.5 \mathrm{~Hz}, 1 \mathrm{H}), 3.79$ (s, 3H), 3.75, (s, 3H), 3.60 (dd, $J=4.5,9.8 \mathrm{~Hz}, 1 \mathrm{H}), 3.46(\mathrm{dd}, J=6.8,9.8 \mathrm{~Hz}, 1 \mathrm{H}), 3.19(\mathrm{~d}, J=22.6 \mathrm{~Hz}, 2 \mathrm{H}), 3.01$ $(\mathrm{dd}, J=4.5,15.9 \mathrm{~Hz}, 1 \mathrm{H}), 2.78(\mathrm{dd}, J=6.8,15.8 \mathrm{~Hz}, 1 \mathrm{H}), 1.04(\mathrm{~s}, 9 \mathrm{H}), 0.85(\mathrm{t}, J=7.5$ $\mathrm{Hz}, 9 \mathrm{H})$ and $0.48(\mathrm{q}, J=7.5 \mathrm{~Hz}, 6 \mathrm{H}) ;{ }^{13} \mathrm{C} \mathrm{NMR}\left(\mathrm{CDCl}_{3}, 75 \mathrm{MHz}\right): \delta 200.5,135.5,133.3$, 129.7, 127.7, 69.2, 67.33, 52.9, 48.8, 43.3, 41.7, 26.8, 19.1, 6.7 and 4.7; HRMS (ESIMS): calcd for $\mathrm{C}_{29} \mathrm{H}_{47} \mathrm{O}_{6} \mathrm{NaSi}_{2} \mathrm{P}[\mathrm{M}+\mathrm{Na}]^{+}: 601.2570$, found: 601.2577 . 
(2S,7R)-1-[1-(tert-Butyl)-1,1-diphenylsilyl]oxy-7-[(4R,5R)-5-ethyl-2-(4-

methoxyphenyl)-1,3-dioxan-4-yl]-2-[(1,1,1-triethylsilyl)oxy]octan-4-one (26) :

Compound 25 (1.74 g, $2.36 \mathrm{mmol})$ was dissolved in EtOAc $(10 \mathrm{~mL}), \mathrm{Pd}-\mathrm{C}(10 \%)$

(70 mg) was added followed by $\mathrm{NH}_{4} \mathrm{OAc}(c a .3 \mathrm{mg}$ ) and subjected to hydrogenation under atmospheric pressure using $\mathrm{H}_{2}$-filled balloon. After $2 \mathrm{~h}$, the reaction mixture was filtered through a short pad of Celite and the filter cake was washed with EtOAc. The filtrate and washings were combined and concentrated in vacuo. Purification by column chromatography $\left(\mathrm{SiO}_{2}, 10 \%\right.$ EtOAc in petroleum ether eluant) afforded pure compound $26(1.66 \mathrm{~g}, 95 \%)$ as a colorless syrup. Optical rotation $[\alpha]_{\mathrm{D}}{ }^{27}=+0.93\left(\right.$ c $\left.3.35, \mathrm{CHCl}_{3}\right)$; IR (neat): $v_{\max } 2956,2877,1712,1247,1112,740$ and $704 \mathrm{~cm}^{-1} ;{ }^{1} \mathrm{H}$ NMR $\left(\mathrm{CDCl}_{3}, 300\right.$ MHz): $\delta$ 7.66-7.54 (m, 4H), 7.41-7.30 (m, 8H), 6.85-6.78 (m, 2H), $5.40(\mathrm{~s}, 1 \mathrm{H}), 4.29-$ $4.12(\mathrm{~m}, 2 \mathrm{H}), 3.89-3.79(\mathrm{~m}, 2 \mathrm{H}), 3.76(\mathrm{~s}, 3 \mathrm{H}), 3.59-3.50(\mathrm{~m}, 1 \mathrm{H}), 3.40(\mathrm{dd}, J=7.3,10.2$ $\mathrm{Hz}, 1 \mathrm{H}), 2.72(\mathrm{dd}, J=4.4,15.4 \mathrm{~Hz}, 1 \mathrm{H}), 2.56-2.40(\mathrm{~m}, 3 \mathrm{H}), 2.09-1.90(\mathrm{~m}, 2 \mathrm{H}), 1.86-1.63$ (m, 2H), 1.48-1.18 (m, 2H), $1.02(\mathrm{~s}, 9 \mathrm{H}), 0.99-0.94(\mathrm{~m}, 3 \mathrm{H}), 0.87-0.77(\mathrm{~m}, 12 \mathrm{H})$ and 0.44 $(\mathrm{q}, J=7.3 \mathrm{~Hz}, 6 \mathrm{H}) ;{ }^{13} \mathrm{C} \mathrm{NMR}\left(\mathrm{CDCl}_{3}, 75 \mathrm{MHz}\right): \delta 209.9,159.7,135.5,133.4,131.2$, $129.6,127.6,127.2,113.5,101.7,84.9,69.3,67.6,55.2,47.5,42.2,37.1,33.4,27.0$, 26.79, 19.1, 16.2, 14.3, 11.9, 6.7, 6.5 and 4.7; HRMS (ESIMS): calcd for $\mathrm{C}_{43} \mathrm{H}_{64} \mathrm{O}_{6} \mathrm{NaSi}_{2}$ $[\mathrm{M}+\mathrm{Na}]^{+}:$755.4139, found: 755.4171.

(2S,7R)-1-[1-(tert-Butyl)-1,1-diphenylsilyl]oxy-7-[(4R,5R)-5-ethyl-2-(4methoxyphenyl)-1,3-dioxan-4-yl]-2-hydroxy-octan-4-one (11):

A solution of compound $26(1.1 \mathrm{~g}, 1.5 \mathrm{mmol})$ in $\mathrm{CH}_{2} \mathrm{Cl}_{2}: \mathrm{MeOH}(4: 1,6 \mathrm{~mL})$ was treated with CSA ( $35 \mathrm{mg}, 0.1 \mathrm{mmol})$ at $0{ }^{\circ} \mathrm{C}$. After being stirred for $10 \mathrm{~min}$, the reaction mixture was quenched with saturated aqueous $\mathrm{NaHCO}_{3}$. Solvent was removed in the 
rotary evaporator and the residue was extracted with EtOAc $(2 \times 30 \mathrm{~mL})$. The combined organic layers were washed with water $(10 \mathrm{~mL})$, brine $(20 \mathrm{~mL})$, dried $\left(\mathrm{Na}_{2} \mathrm{SO}_{4}\right)$ and concentrated in vacuo. Column chromatography $\left(\mathrm{SiO}_{2}, 18-20 \%\right.$ EtOAc in petroleum ether eluant) gave pure compound $\mathbf{1 1}(813 \mathrm{mg}, 87 \%)$ as colorless syrup. Optical rotation $[\alpha]_{\mathrm{D}}{ }^{27}=+11.10\left(\right.$ c 5.5, $\left.\mathrm{CHCl}_{3}\right)$; IR (neat): $v_{\max } 3335(\mathrm{br}), 2930,1728,1462,1111,741$ and $703 \mathrm{~cm}^{-1} ;{ }^{1} \mathrm{H}$ NMR $\left(\mathrm{CDCl}_{3}, 300 \mathrm{MHz}\right): \delta 7.65-7.58(\mathrm{~m}, 4 \mathrm{H}), 7.42-7.31(\mathrm{~m}, 8 \mathrm{H}), 6.85-$ $6.78(\mathrm{~m}, 2 \mathrm{H}), 5.39(\mathrm{~s}, 1 \mathrm{H}), 4.31-4.04(\mathrm{~m}, 2 \mathrm{H}), 3.90-3.78(\mathrm{~m}, 2 \mathrm{H}), 3.75(\mathrm{~s}, 3 \mathrm{H}), 3.56(\mathrm{dd}, J$ $=1.6,3.9 \mathrm{~Hz}, 1 \mathrm{H}), 3.47(\mathrm{dd}, J=1.6,7.8 \mathrm{~Hz}, 1 \mathrm{H}), 2.83(\mathrm{~d}, J=3.9 \mathrm{~Hz}, 1 \mathrm{H}), 2.52-2.40(\mathrm{~m}$, $4 \mathrm{H}), 2.07-1.87(\mathrm{~m}, 2 \mathrm{H}), 1.86-1.63(\mathrm{~m}, 2 \mathrm{H}), 1.41-1.21(\mathrm{~m}, 2 \mathrm{H}), 1.05(\mathrm{~s}, 9 \mathrm{H}), 0.96(\mathrm{t}, J=$ $7.8 \mathrm{~Hz}, 3 \mathrm{H})$ and $0.82(\mathrm{~d}, J=7.0 \mathrm{~Hz}, 3 \mathrm{H}) ;{ }^{13} \mathrm{C} \mathrm{NMR}\left(\mathrm{CDCl}_{3}, 75 \mathrm{MHz}\right): \delta 190.8,159.5$, 135.6, 133.6, 131.9, 129.5, 127.6, 114.3, 113.5, 96.8, 68.4, 67.7, 64.3, 55.5, 44.8, 39.2, 30.2, 26.8, 26.3, 26.0, 20.9, 20.8, 19.2 and 11.2; HRMS (ESIMS): calcd for $\mathrm{C}_{37} \mathrm{H}_{50} \mathrm{O}_{6} \mathrm{NaSi}[\mathrm{M}+\mathrm{Na}]^{+}: 641.3274$, found: 641.3292 .

\section{(2S,4R,7R)-7-[(4R,5R)-5-Ethyl-2-(4-methoxyphenyl)-1,3-dioxan-4-yl]octane-1,2,4-} triol (28):

To a stirred solution of $27(502 \mathrm{mg}, 0.80 \mathrm{mmol})$ in dry THF (2 mL), TBAF (1M solution in THF, $0.9 \mathrm{~mL}, 0.92 \mathrm{mmol}$ ) was added at $0{ }^{\circ} \mathrm{C}$. The reaction mixture was warmed to room temperature and stirred for $4 \mathrm{~h}$. It was quenched with saturated aqueous $\mathrm{NH}_{4} \mathrm{Cl}$ solution, extracted with EtOAc, washed with brine, dried $\left(\mathrm{Na}_{2} \mathrm{SO}_{4}\right)$ and concentrated in vacuo. Purification by column chromatography $\left(\mathrm{SiO}_{2}, 80\right.$ to $100 \%$ EtOAc in petroleum ether eluant) afforded pure 28 (304 mg, 95\%) as colorless oil. Optical rotation $[\alpha]_{\mathrm{D}}^{27}=+16.44\left(\right.$ c $\left.1.8, \mathrm{CHCl}_{3}\right)$; IR (neat): $v_{\max } 3361(\mathrm{br}), 2933,1516,1461,1247$, 1117 and $828 \mathrm{~cm}^{-1} ;{ }^{1} \mathrm{H}$ NMR $\left(\mathrm{CDCl}_{3}, 300 \mathrm{MHz}\right): \delta 7.40-7.36(\mathrm{~m}, 2 \mathrm{H}), 6.89-6.84(\mathrm{~m}, 2 \mathrm{H})$, 
$5.43(\mathrm{~s}, 1 \mathrm{H}), 4.27(\mathrm{~m}, 1 \mathrm{H}), 3.92-3.80(\mathrm{~m}, 3 \mathrm{H}), 3.78(\mathrm{~s}, 3 \mathrm{H}), 3.53-3.46(\mathrm{~m}, 2 \mathrm{H}), 3.40(\mathrm{dd}, J$ $=6.8,11.3 \mathrm{~Hz}, 1 \mathrm{H}), 1.84-1.68(\mathrm{~m}, 3 \mathrm{H}), 1.62-1.49(\mathrm{~m}, 3 \mathrm{H}), 1.49-1.37(\mathrm{~m}, 4 \mathrm{H}), 0.99(\mathrm{t}, J=$ $7.6 \mathrm{~Hz}, 3 \mathrm{H})$ and $0.82(\mathrm{~d}, J=6.8 \mathrm{~Hz}, 3 \mathrm{H}) ;{ }^{13} \mathrm{C} \mathrm{NMR}\left(\mathrm{CDCl}_{3}, 75 \mathrm{MHz}\right): \delta 159.9,131.4$, $127.4,113.6,101.9,85.1,69.6,69.4,68.7,66.8,55.3,39.1,37.0,34.5,33.4,28.9,16.2$, 14.5 and 11.9; HRMS (ESIMS): calcd for $\mathrm{C}_{21} \mathrm{H}_{34} \mathrm{O}_{6} \mathrm{Na}[\mathrm{M}+\mathrm{Na}]^{+}$: 405.2253, found: 405.2247 .

(2S,4R,7R)-1-[1-(tert-Butyl)-1,1-dimethylsilyl]oxy-7-[(4R,5R)-5-ethyl-2-(4methoxyphenyl)-1,3-dioxan-4-yl]octane-2,4-diol (29):

To a stirred solution of $28(304 \mathrm{mg}, 0.79 \mathrm{mmol})$ in $\mathrm{CH}_{2} \mathrm{Cl}_{2}(3 \mathrm{~mL}), \mathrm{Et}_{3} \mathrm{~N}(0.22$ $\mathrm{mL}, 1.59 \mathrm{mmol})$ and $\mathrm{TBSCl}(143 \mathrm{mg}, 0.95 \mathrm{mmol})$ were added sequentially at $0{ }^{\circ} \mathrm{C}$ under nitrogen atmosphere followed by the addition of DMAP $(9.7 \mathrm{mg}, 0.08 \mathrm{mmol})$. The reaction mixture was warmed to room temperature and stirred for $3 \mathrm{~h}$. It was quenched with saturated aqueous $\mathrm{NH}_{4} \mathrm{Cl}$ solution and extracted with EtOAc $(40 \mathrm{~mL})$, washed with brine $(20 \mathrm{~mL})$, dried $\left(\mathrm{Na}_{2} \mathrm{SO}_{4}\right)$ and concentrated in vacuo. Purification by column chromatography ( $\mathrm{SiO}_{2}, 25$ to $30 \%$ EtOAc in petroleum ether eluant) gave pure compound $29(0.3 \mathrm{~g}, 76 \%)$ as clear oil. Optical rotation $[\alpha]_{\mathrm{D}}^{22}=+6.08\left(c 1.2, \mathrm{CHCl}_{3}\right)$; IR (neat): $v_{\max }$ $3450,2930,2857,2338,1615,1462,1249,1119$ and $834 \mathrm{~cm}^{-1} ;{ }^{1} \mathrm{H}$ NMR $\left(\mathrm{CDCl}_{3}, 400\right.$ MHz): $\delta 7.39(\mathrm{~d}, J=8.7 \mathrm{~Hz}, 2 \mathrm{H}), 6.87(\mathrm{~d}, J=8.7 \mathrm{~Hz}, 2 \mathrm{H}), 5.43(\mathrm{~s}, 1 \mathrm{H}), 4.27(\mathrm{~m}, 1 \mathrm{H})$, 3.98-3.82 (m, 4H), 3.79 (s, 3H), $3.58(\mathrm{dd}, J=4.4,10.2 \mathrm{~Hz}, 1 \mathrm{H}), 3.45(\mathrm{dd}, J=7.3,10.2$ Hz, 1H), 1.87-1.66 (m, 3H), 1.63-1.47 (m, 4H), 1.45-1.27 (m, 3H), 0.99 (t, $J=7.3 \mathrm{~Hz}$, 3H), $0.89(\mathrm{~s}, 9 \mathrm{H}), 0.83(\mathrm{~d}, J=6.5 \mathrm{~Hz}, 3 \mathrm{H})$ and $0.06(\mathrm{~s}, 6 \mathrm{H}) ;{ }^{13} \mathrm{C} \mathrm{NMR}\left(\mathrm{CDCl}_{3}, 100\right.$ MHz): $\delta 159.8,131.5,127.3,113.5,101.8,85.0,69.5,68.9,67.1,55.2,38.9,37.0,34.6$, $33.6,29.0,25.8,18.3,16.2,14.3,12.0$ and -5.4 . 
HRMS (ESIMS): calcd for $\mathrm{C}_{27} \mathrm{H}_{48} \mathrm{O}_{6} \mathrm{NaSi}[\mathrm{M}+\mathrm{Na}]^{+}:$519.3117, found: 519.3136 .

tert-Butyl((2S,4R,7R)-7-[(4R,5R)-5-ethyl-2-(4-methoxyphenyl)-1,3-dioxan-4-yl]-2,4di[(1,1,1-triisopropylsilyl)oxy]octyloxy)dimethylsilane (10):

To a stirred solution of compound $29(300 \mathrm{mg}, 0.6 \mathrm{mmol})$ in $\mathrm{CH}_{2} \mathrm{Cl}_{2}(2 \mathrm{~mL})$ at 0 ${ }^{\circ} \mathrm{C}$, 2,6-lutidine $(0.43 \mathrm{~mL}, 2.42 \mathrm{mmol})$ and TIPSOTf $(0.36 \mathrm{~mL}, 1.33 \mathrm{mmol})$ were added sequentially. After $15 \mathrm{~min}$, reaction was quenched with saturated $\mathrm{NH}_{4} \mathrm{Cl}$ solution (10 $\mathrm{mL})$, the reaction mixture was extracted with EtOAc $(2 \times 30 \mathrm{~mL})$, washed with saturated aqueous $\mathrm{CuSO}_{4}$ solution $(10 \mathrm{~mL})$, brine $(10 \mathrm{~mL})$, dried $\left(\mathrm{Na}_{2} \mathrm{SO}_{4}\right)$ and concentrated in vacuo. Purification by column chromatography $\left(\mathrm{SiO}_{2}, 2-5 \%\right.$ EtOAc in petroleum ether eluant) afforded pure compound 10 (460 mg, 95\%) as colorless liquid. Optical rotation $[\alpha]_{\mathrm{D}}^{22}=+10.66\left(\right.$ c 2.25, $\left.\mathrm{CHCl}_{3}\right)$; IR (neat): $v_{\max } 2946,2864,2359,1464,1215,759$ and

$671 \mathrm{~cm}^{-1} ;{ }^{1} \mathrm{H} \mathrm{NMR}\left(\mathrm{CDCl}_{3}, 400 \mathrm{MHz}\right): \delta 7.39(\mathrm{~d}, J=8.9 \mathrm{~Hz}, 2 \mathrm{H}), 6.85(\mathrm{~d}, J=8.9 \mathrm{~Hz}$, 2H), $5.43(\mathrm{~s}, 1 \mathrm{H}), 4.28(\mathrm{~m}, 1 \mathrm{H}), 4.00(\mathrm{dd}, J=4.0,6.9 \mathrm{~Hz}, 1 \mathrm{H}) 3.91-3.82(\mathrm{~m}, 2 \mathrm{H}), 3.79(\mathrm{~s}$, $3 \mathrm{H}), 3.58(\mathrm{dd}, J=4.0,9.4 \mathrm{~Hz}, 1 \mathrm{H}), 3.50-3.44(\mathrm{~m}, 2 \mathrm{H}), 1.94-1.76(\mathrm{~m}, 2 \mathrm{H}), 1.74-1.64(\mathrm{~m}$, $2 \mathrm{H}), 1.61-1.54(\mathrm{~m}, 1 \mathrm{H}), 1.53-1.43(\mathrm{~m}, 2 \mathrm{H}), 1.36-1.31(\mathrm{~m}, 2 \mathrm{H}), 1.20(\mathrm{~m}, 1 \mathrm{H}), 1.08-1.01$ $(\mathrm{m}, 42 \mathrm{H}), 0.99(\mathrm{t}, J=7.9 \mathrm{~Hz}, 3 \mathrm{H}), 0.86(\mathrm{~s}, 9 \mathrm{H}), 0.81(\mathrm{~d}, J=6.5 \mathrm{~Hz}, 3 \mathrm{H})$ and $0.02(\mathrm{~s}, 6 \mathrm{H})$; ${ }^{13} \mathrm{C} \mathrm{NMR}\left(\mathrm{CDCl}_{3}, 100 \mathrm{MHz}\right): \delta 159.6,131.8,127.3,113.4,101.7,84.9,71.4,70.3,69.4$, $68.3,55.2,43.8,37.1,34.5,34.1,27.8,25.9,18.3,18.2,18.0,17.7,16.3,14.1,12.8,12.7$, 12.3, 12.0 and -5.5; HRMS (ESIMS): calcd for $\mathrm{C}_{45} \mathrm{H}_{88} \mathrm{O}_{6} \mathrm{NaSi}_{3}[\mathrm{M}+\mathrm{Na}]^{+}:$831.5786, found: 831.5756 .

(2R,3R,4R,7R,9S)-10-[1-(tert-Butyl)-1,1-dimethylsilyl]oxy-2-ethyl-3-[(4methoxybenzyl)oxy]-4-methyl-7,9-di[(1,1,1-triisopropylsilyl)oxy]-decan-1-ol (30): 
To a solution of compound $10(460 \mathrm{mg}, 0.57 \mathrm{mmol})$ in dry $\mathrm{CH}_{2} \mathrm{Cl}_{2}(4 \mathrm{~mL})$ at $40^{\circ} \mathrm{C}$, DIBAL-H (1.4 M solution in toluene, $\left.1.6 \mathrm{~mL}, 2.28 \mathrm{mmol}\right)$ was added portion wise with stirring under nitrogen atmosphere. After stirring for $0.5 \mathrm{~h}$ at the same temperature, the reaction mixture was slowly brought to $0{ }^{\circ} \mathrm{C}$ and stirred for $2 \mathrm{~h}$ and then quenched with dry $\mathrm{MeOH}(3 \mathrm{~mL})$ at $-78{ }^{\circ} \mathrm{C}$, stirred for $0.5 \mathrm{~h}$ at the same temperature, then added potassium sodium tartarate, stirred at room temperature for $1 \mathrm{~h}$ and extracted with EtOAc $(2 \times 30 \mathrm{~mL})$. The combined organic extracts were washed with brine $(20 \mathrm{~mL})$, dried $\left(\mathrm{Na}_{2} \mathrm{SO}_{4}\right)$ and concentrated in vacuo. Purification of the residue by column chromatography $\left(\mathrm{SiO}_{2}, 7 \%\right.$ EtOAc in petroleum ether) provided compound $30(422 \mathrm{mg}$, $92 \%$ ) as colorless oil. Optical rotation $[\alpha]_{\mathrm{D}}{ }^{27}=-0.33$ (c 1.82, $\mathrm{CHCl}_{3}$ ); IR (neat): $v_{\max }$ 2927(br), 2866, 1215, 759, and $670 \mathrm{~cm}^{-1} ;{ }^{1} \mathrm{H}$ NMR ( $\left.\mathrm{CDCl}_{3}, 400 \mathrm{MHz}\right): \delta$ 7.28-7.19 (m, 2H), 6.86-6.79 (m, 2H), $4.47(\mathrm{ABq}, J=10.7 \mathrm{~Hz}, 2 \mathrm{H}), 4.01(\mathrm{brm}, 1 \mathrm{H}), 3.81(\mathrm{~m}, 1 \mathrm{H}), 3.76$ (s, 3H), $3.64(\mathrm{~d}, J=4.5 \mathrm{~Hz}, 2 \mathrm{H}), 3.57$ (dd, $J=4.0,9.4 \mathrm{~Hz}, 1 \mathrm{H}), 3.47$ (m, 1H), 3.35 (m, 1H), 1.87-1.53 (m, 7H), 1.52-1.37 (m, 2H), $1.29(\mathrm{~m}, 1 \mathrm{H}), 1.10-0.99(\mathrm{~m}, 45 \mathrm{H}), 0.93-0.82$ (m, 12H) and $0.01(\mathrm{~s}, 6 \mathrm{H})$; HRMS (ESIMS): calcd for $\mathrm{C}_{45} \mathrm{H}_{90} \mathrm{O}_{6} \mathrm{NaSi}_{3}[\mathrm{M}+\mathrm{Na}]^{+}$: 833.5942, found: 833.5925 .

\section{tert-Butyl((2S,4R,7R,8R,9R)-9-ethyl-8-[(4-methoxybenzyl)oxy]-7-methyl-2,4-} di[(1,1,1-triisopropylsilyl)oxy]-10-undecenyloxy)dimethylsilane (31):

To a stirred solution of $\mathbf{3 0}(422 \mathrm{mg}, 0.52 \mathrm{mmol})$ in $\mathrm{CH}_{2} \mathrm{Cl}_{2}(3 \mathrm{~mL}), \mathrm{NaHCO}_{3}(148$ $\mathrm{mg}, 1.04 \mathrm{mmol}$ ) was added at $0{ }^{\circ} \mathrm{C}$, followed by Dess-Martin periodinane (DMP) (332 $\mathrm{mg}, 0.78 \mathrm{mmol}$ ) under nitrogen atmosphere. The reaction mixture was allowed to come to room temperature and stirred for $30 \mathrm{~min}$. Saturated $\mathrm{Na}_{2} \mathrm{~S}_{2} \mathrm{O}_{3}(10 \mathrm{~mL})$ and $\mathrm{NaHCO}_{3}(2$ $\mathrm{mL}$ ) were added, and the biphasic mixture was stirred for $15 \mathrm{~min}$ and extracted with 
hexane. The organic phase was washed with water $(20 \mathrm{~mL})$, brine $(20 \mathrm{~mL})$, dried $\left(\mathrm{Na}_{2} \mathrm{SO}_{4}\right)$ and concentrated in vacuo. The aldehyde $\left(\mathrm{R}_{f}=0.5,3 \%\right.$ EtOAc in petroleum ether), thus obtained, was directly used, after flash chromatography, for the next reaction without any further characterization.

Sodamide (244 mg, $6.26 \mathrm{mmol}$ ) was added to a suspension of methyltriphenylphoshonium iodide $(844 \mathrm{mg}, 2.08 \mathrm{mmol})$ in dry ether $(10 \mathrm{~mL})$ at $0{ }^{\circ} \mathrm{C}$. The mixture was allowed to come to room temperature and stirred for $6 \mathrm{~h}$. The methylenetriphenylphosphorane, thus obtained, was added to a solution of aldehyde in ether $(4 \mathrm{~mL})$ at $0{ }^{\circ} \mathrm{C}$. After $10 \mathrm{~min}$, the reaction mixture was quenched with saturated aqueous $\mathrm{NH}_{4} \mathrm{Cl}$ solution. Crude reaction mixture was extracted with ether $(50 \mathrm{~mL})$, washed with water $(20 \mathrm{~mL})$, brine $(15 \mathrm{~mL})$ dried $\left(\mathrm{Na}_{2} \mathrm{SO}_{4}\right)$ and concentrated in vacuo. Purification by column chromatography $\left(\mathrm{SiO}_{2}, 2\right.$ to $4 \%$ EtOAc in petroleum ether eluant) gave pure compound $31(402 \mathrm{mg}, 96 \%)$ as clear oil. Optical rotation $[\alpha]_{\mathrm{D}}^{23}=+0.7$ (c 2.0, $\left.\mathrm{CHCl}_{3}\right)$; IR (neat): $v_{\max } 2944,2865,2362,2339,1651,1513,1462,1379$ and $1249 \mathrm{~cm}^{-1} ;{ }^{1} \mathrm{H}$ NMR $\left(\mathrm{CDCl}_{3}, 400 \mathrm{MHz}\right): \delta 7.21(\mathrm{~d}, J=8.6 \mathrm{~Hz}, 2 \mathrm{H}), 6.80(\mathrm{~d}, J=8.6 \mathrm{~Hz}, 2 \mathrm{H}), 5.54(\mathrm{~m}, 1 \mathrm{H})$, 5.00-4.91 (m, 2H), $4.43(\mathrm{ABq}, J=10.1,10.9 \mathrm{~Hz}, 2 \mathrm{H}), 3.94(\mathrm{~m}, 1 \mathrm{H}), 3.80(\mathrm{~m}, 1 \mathrm{H}), 3.75$ (s, 3H), 3.53 (dd, $J=5.4,9.3 \mathrm{~Hz}, 2 \mathrm{H}), 3.44$ (dd, $J=5.4,10.1 \mathrm{~Hz}, 1 \mathrm{H}), 3.02(\mathrm{~m}, 1 \mathrm{H}), 2.12$ (m, 1H), 1.79-1.70 (m, 3H), 1.67-1.52 (m, 3H), $1.36(\mathrm{~m}, 1 \mathrm{H}), 1.17(\mathrm{~m}, 1 \mathrm{H}), 1.06-0.97(\mathrm{~m}$, 42H), $0.90(\mathrm{~d}, J=7.0 \mathrm{~Hz}, 3 \mathrm{H}), 0.83(\mathrm{~m}, 12 \mathrm{H}), 0.77(\mathrm{t}, J=7.9 \mathrm{~Hz}, 3 \mathrm{H})$ and $0.02(\mathrm{~s}, 6 \mathrm{H})$; ${ }^{13} \mathrm{C}$ NMR $\left(\mathrm{CDCl}_{3}, 100 \mathrm{MHz}\right): \delta 140.6,131.4,128.9,115.6,113.6,87.5,74.3,71.4,70.3$, $68.3,55.2,49.3,43.7,36.6,35.8,26.4,25.9,22.5,18.3,18.2,16.9,12.8,12.7,11.8$ and 5.4; HRMS (ESIMS): calcd for $\mathrm{C}_{46} \mathrm{H}_{90} \mathrm{O}_{5} \mathrm{NaSi}_{3}[\mathrm{M}+\mathrm{Na}]^{+}:$829.5993, found: 829.5963 . 
(3R,4R,5R,8R,10S)-11-[1-(tert-Butyl)-1,1-dimethylsilyl]oxy-3-ethyl-5-methyl-8,10di[(1,1,1-triisopropylsilyl)oxy]-1-undecen-4-ol (32):

To a stirred solution of 31 (400 mg, $0.49 \mathrm{mmol})$ in $\mathrm{CHCl}_{3}: \mathrm{pH} 7$ Phosphate Buffer (20:1, $5 \mathrm{~mL})$, DDQ $(225 \mathrm{mg}, 0.993 \mathrm{mmol})$ was added at $0{ }^{\circ} \mathrm{C}$. The reaction mixture was then allowed to come to room temperature and stirred for $30 \mathrm{~min}$. The reaction mixture was quenched with saturated $\mathrm{NaHCO}_{3}(10 \mathrm{~mL})$ and extracted with ether $(60 \mathrm{~mL})$, washed with water $(30 \mathrm{~mL})$, brine $(30 \mathrm{~mL})$, dried $\left(\mathrm{Na}_{2} \mathrm{SO}_{4}\right)$ and concentrated in vacuo. Purification by column chromatography $\left(\mathrm{SiO}_{2}, 7\right.$ to $9 \%$ EtOAc in petroleum ether eluant) gave pure compound $32(310 \mathrm{mg}, 91 \%)$ as clear oil. Optical rotation $[\alpha]_{\mathrm{D}}{ }^{27}=+6.37(\mathrm{c}$ 2.12, $\mathrm{CHCl}_{3}$ ); IR (neat): $v_{\max } 3020$ (br), 2867, 1731, 1249, 1217, 1046, 759, and $671 \mathrm{~cm}^{-}$

${ }^{1} ;{ }^{1} \mathrm{H} \mathrm{NMR}\left(\mathrm{CDCl}_{3}, 400 \mathrm{MHz}\right): \delta 5.57(\mathrm{~m}, 1 \mathrm{H}), 5.11-4.98(\mathrm{~m}, 2 \mathrm{H}), 4.01(\mathrm{~m}, 1 \mathrm{H}), 3.84(\mathrm{~m}$, 1H), $3.59(\mathrm{dd}, J=4.7,10.1 \mathrm{~Hz}, 1 \mathrm{H}), 3.49(\mathrm{dd}, J=6.2,10.1 \mathrm{~Hz}, 1 \mathrm{H}), 3.26(\mathrm{~m}, 1 \mathrm{H}), 2.07$ (m, 1H), 1.86-1.76 (m, 2H), 1.74-1.66 (m, 3H), 1.64-1.55 (m, 3H), $1.40(\mathrm{~m}, 1 \mathrm{H}), 1.09-$ $1.03(\mathrm{~m}, 42 \mathrm{H}), 0.91(\mathrm{~d}, J=6.2 \mathrm{~Hz}, 3 \mathrm{H}), 0.88(\mathrm{~s}, 9 \mathrm{H}), 0.84(\mathrm{t}, J=7.8 \mathrm{~Hz}, 3 \mathrm{H})$ and $0.04(\mathrm{~s}$, $6 \mathrm{H}) ;{ }^{13} \mathrm{C} \mathrm{NMR}\left(\mathrm{CDCl}_{3}, 100 \mathrm{MHz}\right): \delta 139.8,116.1,79.1,71.4,70.1,68.3,49.5,43.6,36.1$, 35.2, 25.9, 24.5, 22.0, 18.3, 18.2, 18.0, 16.9, 12.8, 12.7, 11.7 and -5.5; HRMS (ESIMS): calcd for $\mathrm{C}_{38} \mathrm{H}_{82} \mathrm{O}_{4} \mathrm{NaSi}_{3}[\mathrm{M}+\mathrm{Na}]^{+}:$709.5412, found: 709.5405 .

(3R,4R,5R,8R,10S)-11-(tert-Butyl-dimethyl-silyloxy)-3-ethyl-5-methyl-8,10-ditriisopropylsilanyloxy-undec-1-en-4-ol acrylate (9):

To a stirred solution of $32(250 \mathrm{mg}, 0.36 \mathrm{mmol})$ in $\mathrm{CH}_{2} \mathrm{Cl}_{2}(3 \mathrm{~mL}), \mathrm{Et}_{3} \mathrm{~N}(0.25$ $\mathrm{mL}, 1.82 \mathrm{mmol})$ and acryloyl chloride $(0.06 \mathrm{~mL}, 0.73 \mathrm{mmol})$ were added sequentially at $0{ }^{\circ} \mathrm{C}$ under nitrogen atmosphere. The reaction mixture was warmed to room temperature and stirred for $0.5 \mathrm{~h}$. It was quenched with water and extracted with EtOAc, washed with 
saturated aqueous $\mathrm{NaHCO}_{3}$ solution $(5 \mathrm{~mL})$, brine $(5 \mathrm{~mL})$, dried $\left(\mathrm{Na}_{2} \mathrm{SO}_{4}\right)$ and concentrated in vacuo. Purification by column chromatography $\left(\mathrm{SiO}_{2}, 2\right.$ to $3 \%$ EtOAc in petroleum ether eluant) gave pure compound $9(172 \mathrm{mg}, 65 \%)$ as clear oil. Optical rotation $[\alpha]_{\mathrm{D}}^{24}=+0.99\left(c 4.45, \mathrm{CHCl}_{3}\right)$; IR (neat): $v_{\max } 2924,2864,1721,1216,759$ and $671 \mathrm{~cm}^{-1} ;{ }^{1} \mathrm{H} \mathrm{NMR}\left(\mathrm{CDCl}_{3}, 400 \mathrm{MHz}\right): \delta 6.37(\mathrm{dd}, J=1.5,17.2 \mathrm{~Hz}, 1 \mathrm{H}), 6.10(\mathrm{dd}, J=$ 10.3, 17.2 Hz, 1H), $5.81(\mathrm{dd}, J=1.5,10.3 \mathrm{~Hz}), 5.47(\mathrm{~m}, 1 \mathrm{H}), 5.40(\mathrm{dd}, J=3.7,10.1 \mathrm{~Hz}$, 1H), $5.02(\mathrm{dd}, J=1.6,11.2 \mathrm{~Hz}), 4.88(\mathrm{dd}, J=3.1,8.7 \mathrm{~Hz}, 1 \mathrm{H}), 4.01(\mathrm{~m}, 1 \mathrm{H}), 3.81(\mathrm{~m}$, 1H), $3.60(\mathrm{dd}, J=4.4,9.8 \mathrm{~Hz}, 1 \mathrm{H}), 3.48(\mathrm{dd}, J=5.8,9.8 \mathrm{~Hz}, 1 \mathrm{H}), 2.25(\mathrm{~m}, 1 \mathrm{H}), 1.86-$ $0.99(\mathrm{~m}, 45 \mathrm{H}), 0.90-0.80(\mathrm{~m}, 12 \mathrm{H})$ and $0.03(\mathrm{~s}, 6 \mathrm{H}) ;{ }^{13} \mathrm{C} \mathrm{NMR}\left(\mathrm{CDCl}_{3}, 100 \mathrm{MHz}\right): \delta$ $166.2,138.3,130.4,128.7,117.0,80.1,71.4,70.0,68.3,47.9,43.7,35.1,25.9,24.2,22.8$, 18.3, 18.2, 17.9, 16.9, 12.7, 12.7, 11.4 and -5.5; HRMS (ESIMS): calcd for $\mathrm{C}_{41} \mathrm{H}_{84} \mathrm{O}_{5} \mathrm{NaSi}_{3}[\mathrm{M}+\mathrm{Na}]^{+}:$763.5524, found: 763.5527 .

(5R,6R)-5-Ethyl-6-((1R,4R,6S)-7-hydroxy-1-methyl-4,6-di-1,1,1-triisopropylsilyloxyheptyl)-5,6-dihydro-2H-2-pyranone (34):

To a stirred solution of compound 33 (35 mg, $0.049 \mathrm{mmol})$ in dry $\mathrm{CH}_{2} \mathrm{Cl}_{2}: \mathrm{MeOH}$ (4:1, $1 \mathrm{~mL})$, CSA $(1.1 \mathrm{mg}, 0.005 \mathrm{mmol})$ was added at $0{ }^{\circ} \mathrm{C}$ under nitrogen atmosphere and stirred for $24 \mathrm{~h}$ at the same temperature. Then, it was quenched with saturated aqueous $\mathrm{NaHCO}_{3}$ solution, extracted with EtOAc $(15 \mathrm{~mL})$, washed with brine $(10 \mathrm{~mL})$, dried $\left(\mathrm{Na}_{2} \mathrm{SO}_{4}\right)$ and concentrated in vacuo. Purification by column chromatography $\left(\mathrm{SiO}_{2}, 20\right.$ to $25 \%$ EtOAc in petroleum ether eluant) eluted compound 34 (20 mg, 80\% based on recovered starting material) as colorless liquid. Optical rotation $[\alpha]_{\mathrm{D}}{ }^{27}=-52.5(c$ 1.50, $\left.\mathrm{CHCl}_{3}\right) ; \mathrm{IR}$ (neat): $v_{\max } 3476,2911,1721,1520$ and $925 \mathrm{~cm}^{-1} ;{ }^{1} \mathrm{H} \mathrm{NMR}\left(\mathrm{CDCl}_{3}, 400\right.$ MHz): $\delta 7.05(\mathrm{dd}, J=6.2,9.4 \mathrm{~Hz}, 1 \mathrm{H}), 6.03(\mathrm{~d}, J=9.4 \mathrm{~Hz}, 1 \mathrm{H}), 3.97(\mathrm{dd}, J=3.1,10.9$ 
$\mathrm{Hz}), 3.93(\mathrm{~m}, 1 \mathrm{H}), 3.85(\mathrm{~m}, 1 \mathrm{H}), 3.65(\mathrm{dd}, J=3.1,10.9 \mathrm{~Hz}), 3.48(\mathrm{dd}, J=3.1,10.1 \mathrm{~Hz}$, 1H), $2.31(\mathrm{~m}, 1 \mathrm{H}), 1.97-1.85(\mathrm{~m}, 2 \mathrm{H}), 1.83-1.75(\mathrm{~m}, 2 \mathrm{H}), 1.72-1.61(\mathrm{~m}, 2 \mathrm{H}), 1.56-1.42$ $(\mathrm{m}, 3 \mathrm{H}), 1.08-1.03(\mathrm{~m}, 42 \mathrm{H}), 0.95(\mathrm{t}, J=7.8 \mathrm{~Hz}, 3 \mathrm{H})$ and $0.88(\mathrm{~d}, J=6.2 \mathrm{~Hz}, 3 \mathrm{H}) ;{ }^{13} \mathrm{C}$ NMR $\left(\mathrm{CDCl}_{3}, 100 \mathrm{MHz}\right): \delta 164.4,150.9,121.0,84.2,70.9,70.3,66.1,41.8,36.6,34.1$, 33.7, 27.9, 20.1, 18.2, 18.1, 14.8, 12.8, 12.4 and 11.0; HRMS (ESIMS): calcd for $\mathrm{C}_{33} \mathrm{H}_{66} \mathrm{O}_{5} \mathrm{NaSi}_{2}[\mathrm{M}+\mathrm{Na}]^{+}:$621.4346, found: 621.4346 . 


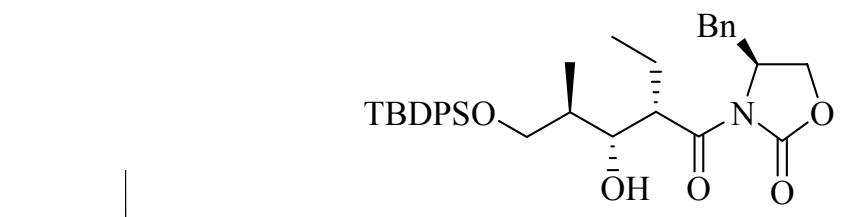

17
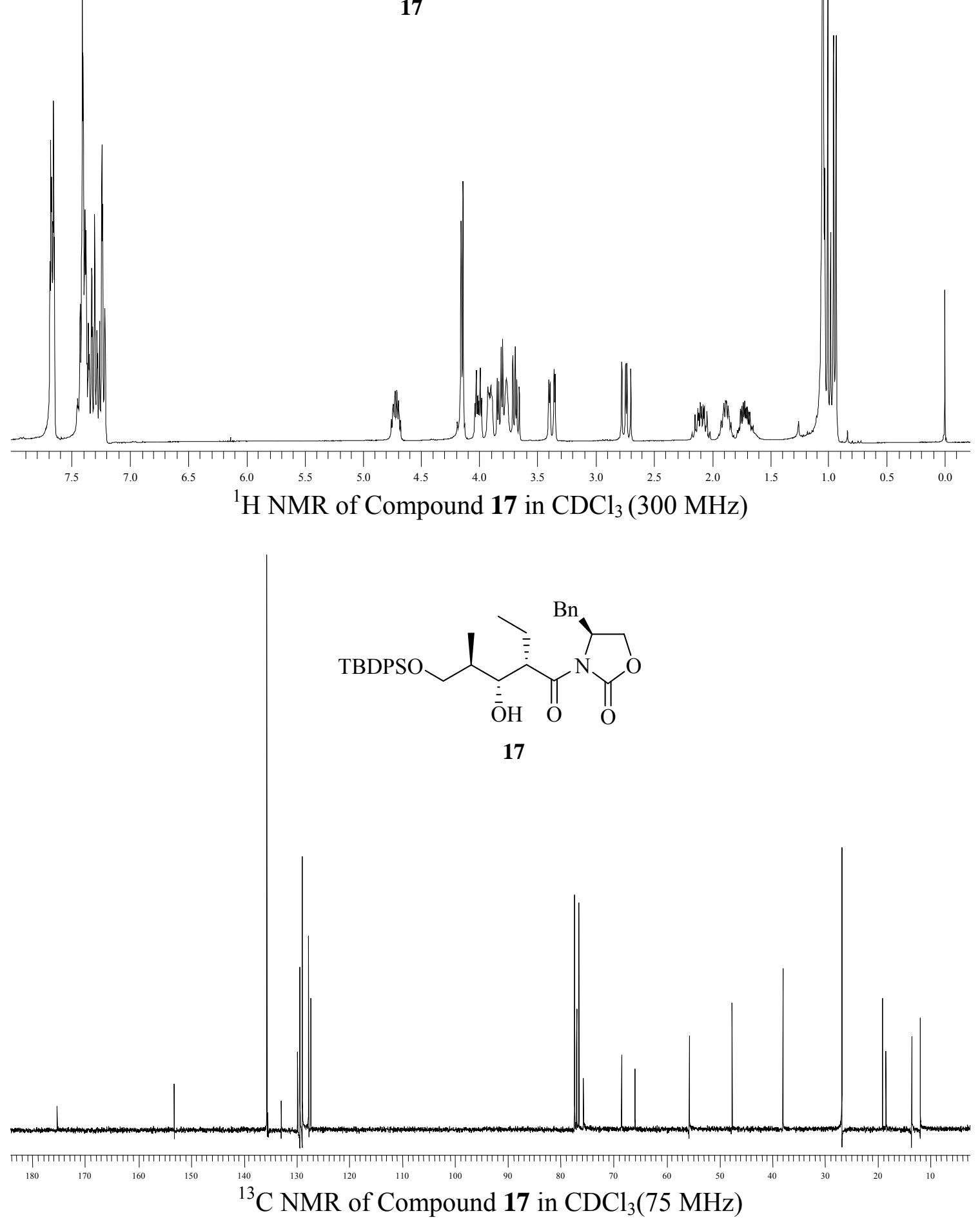


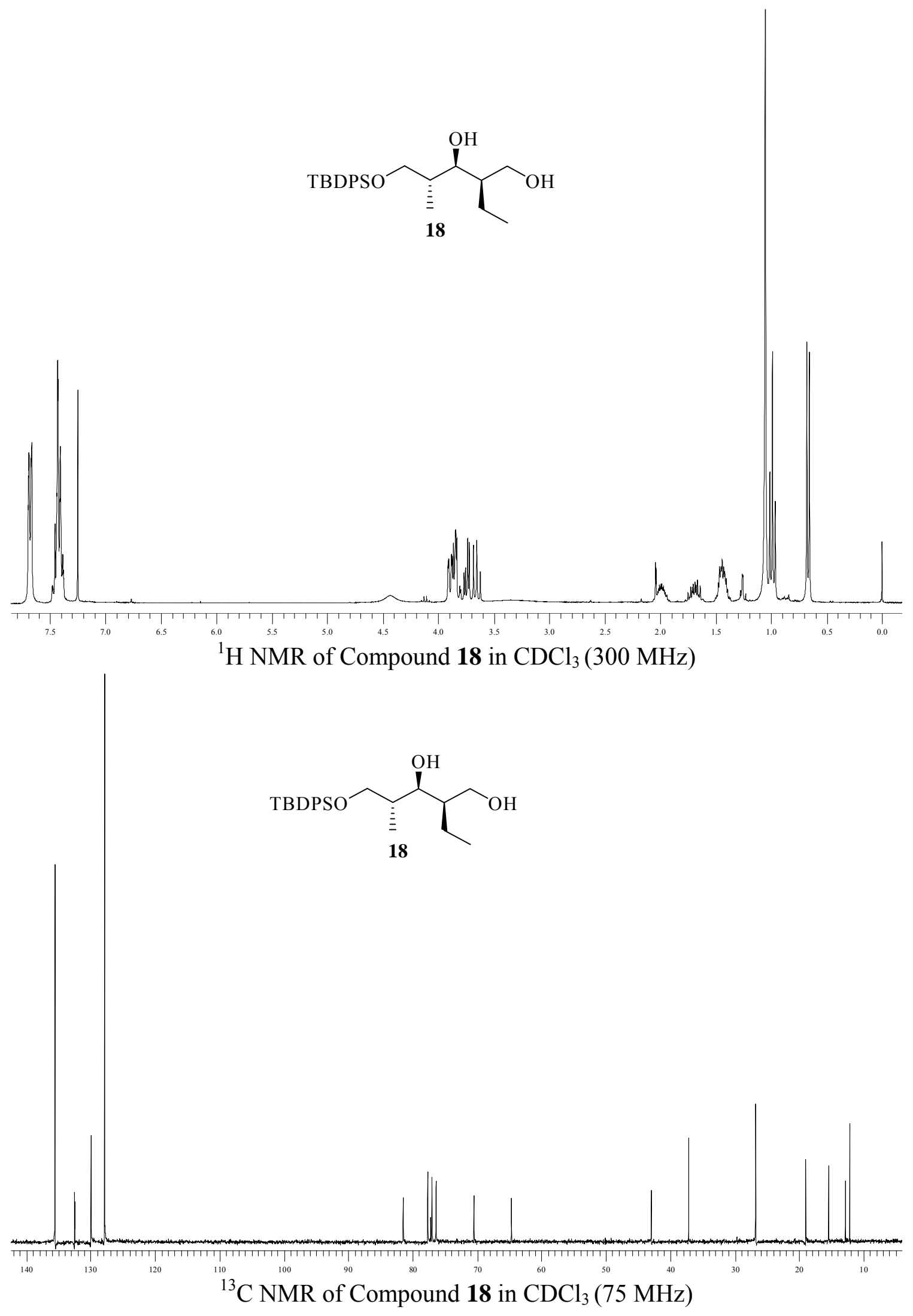




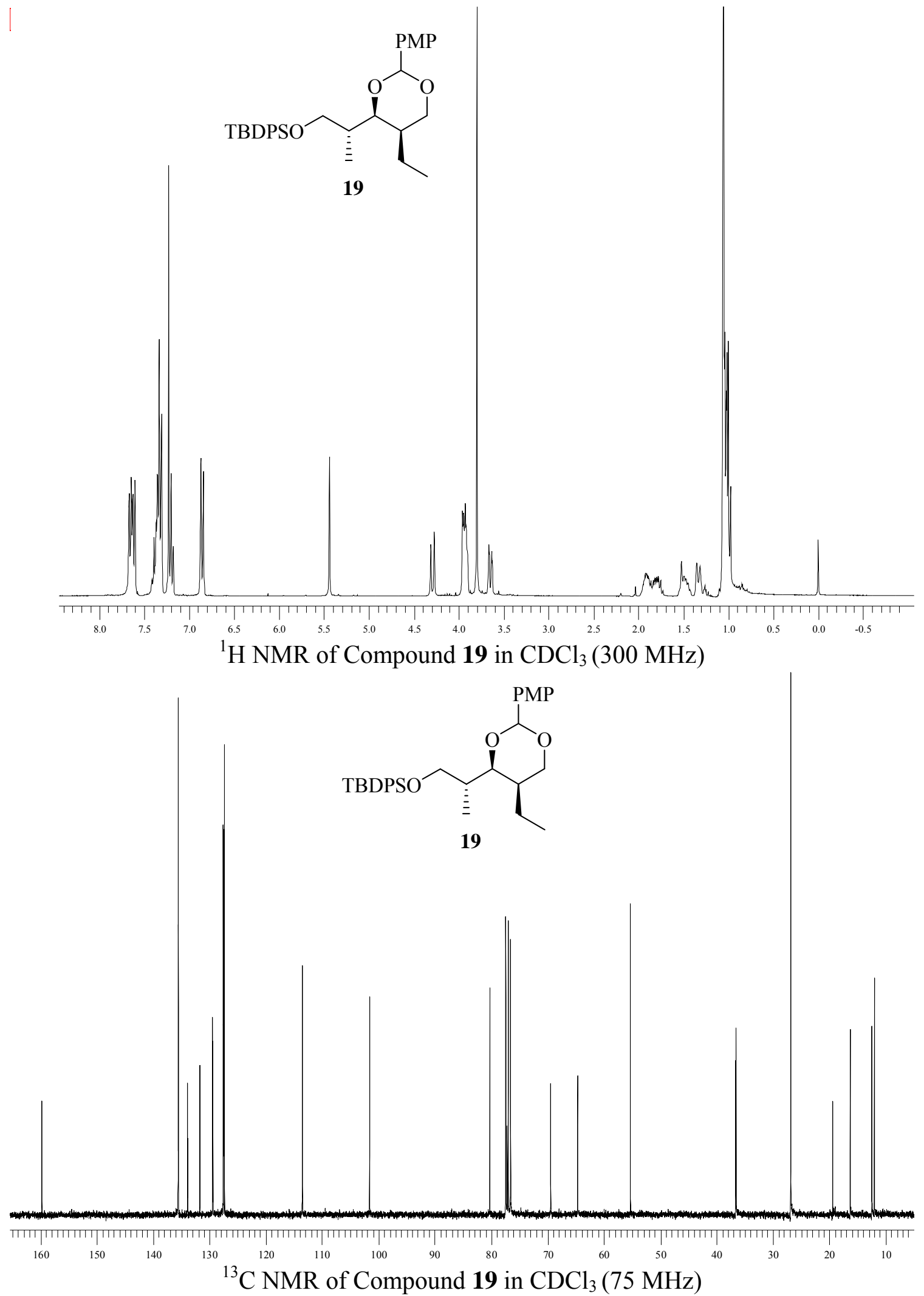



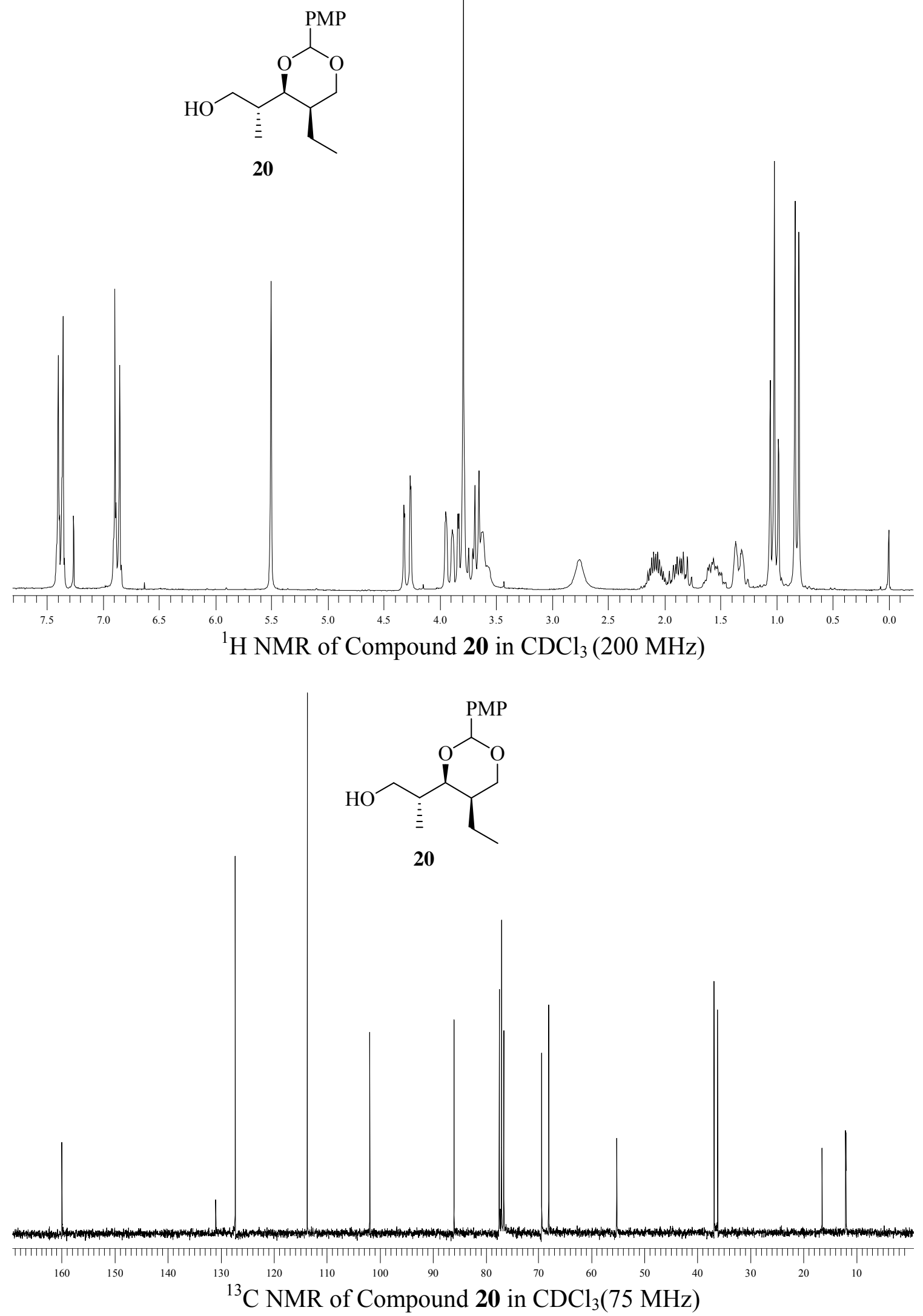


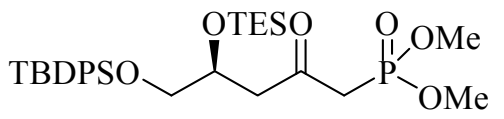

12
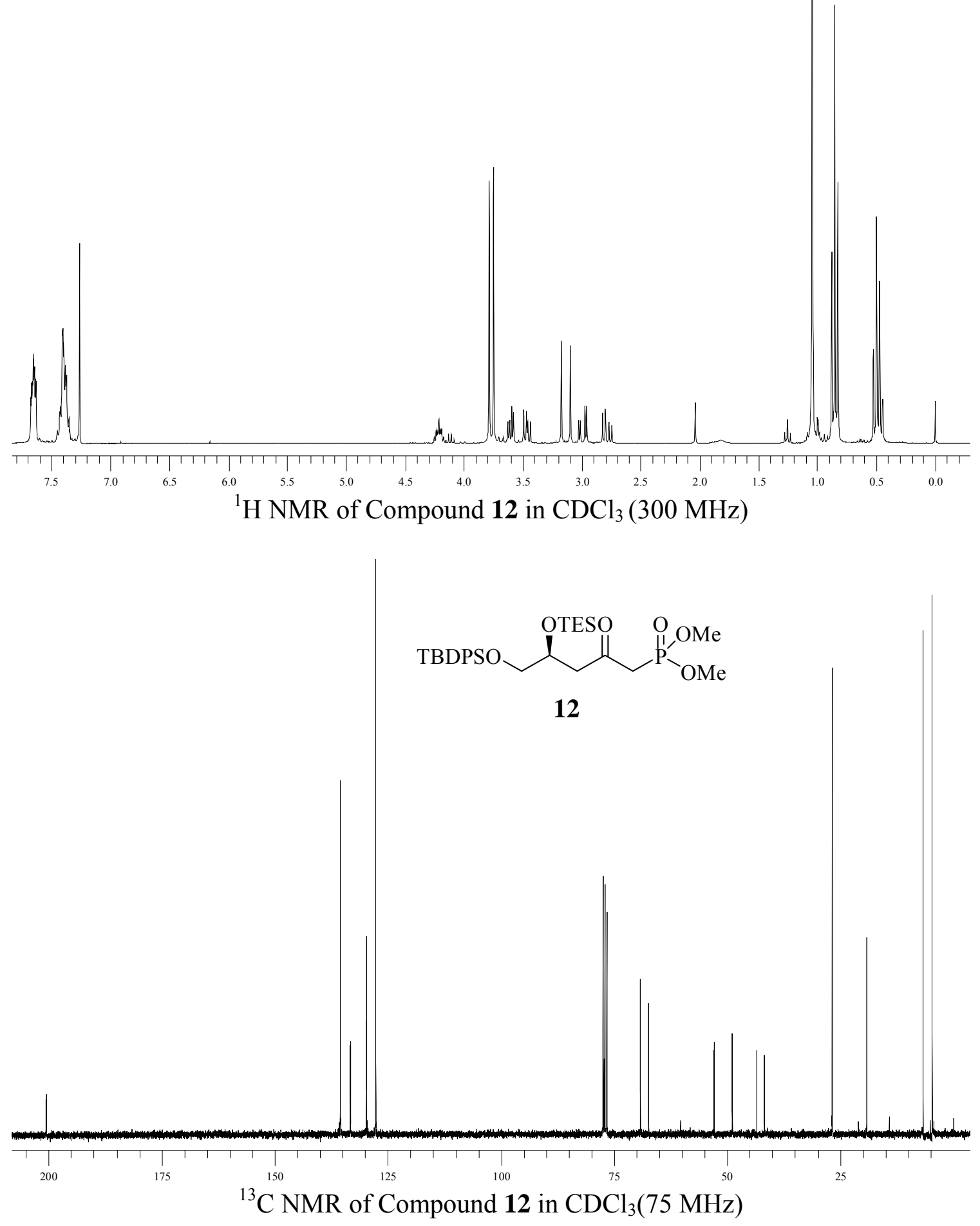

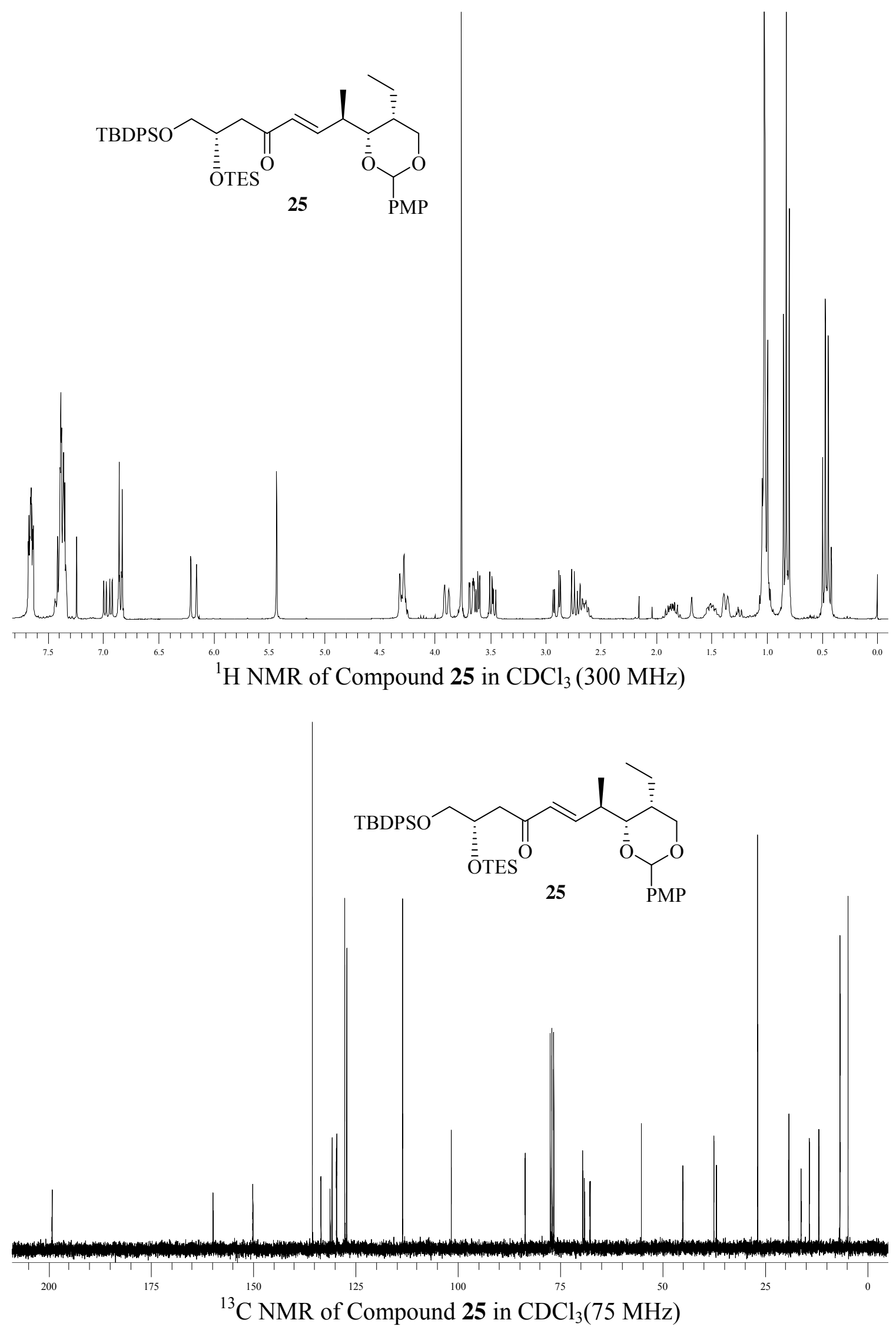

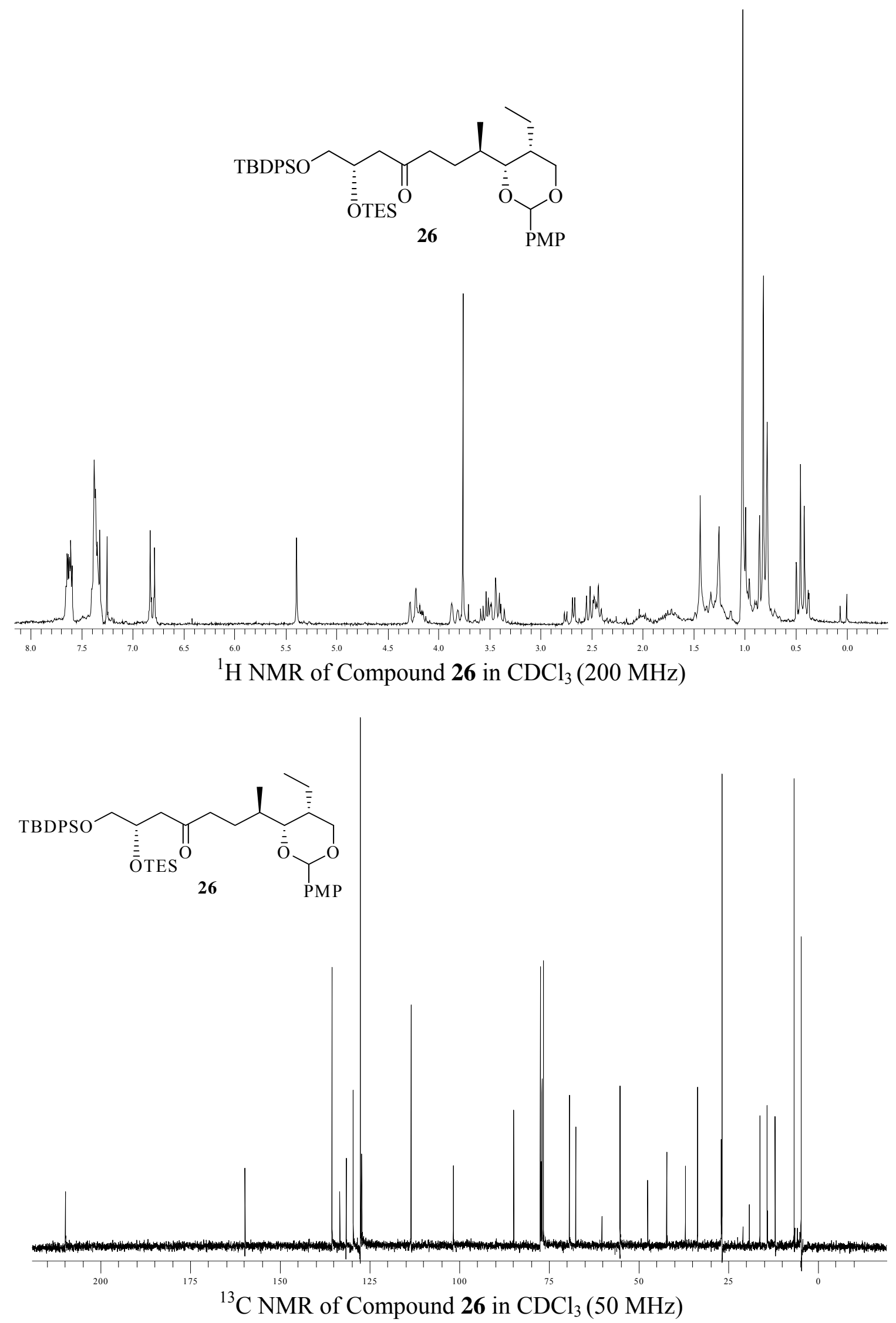


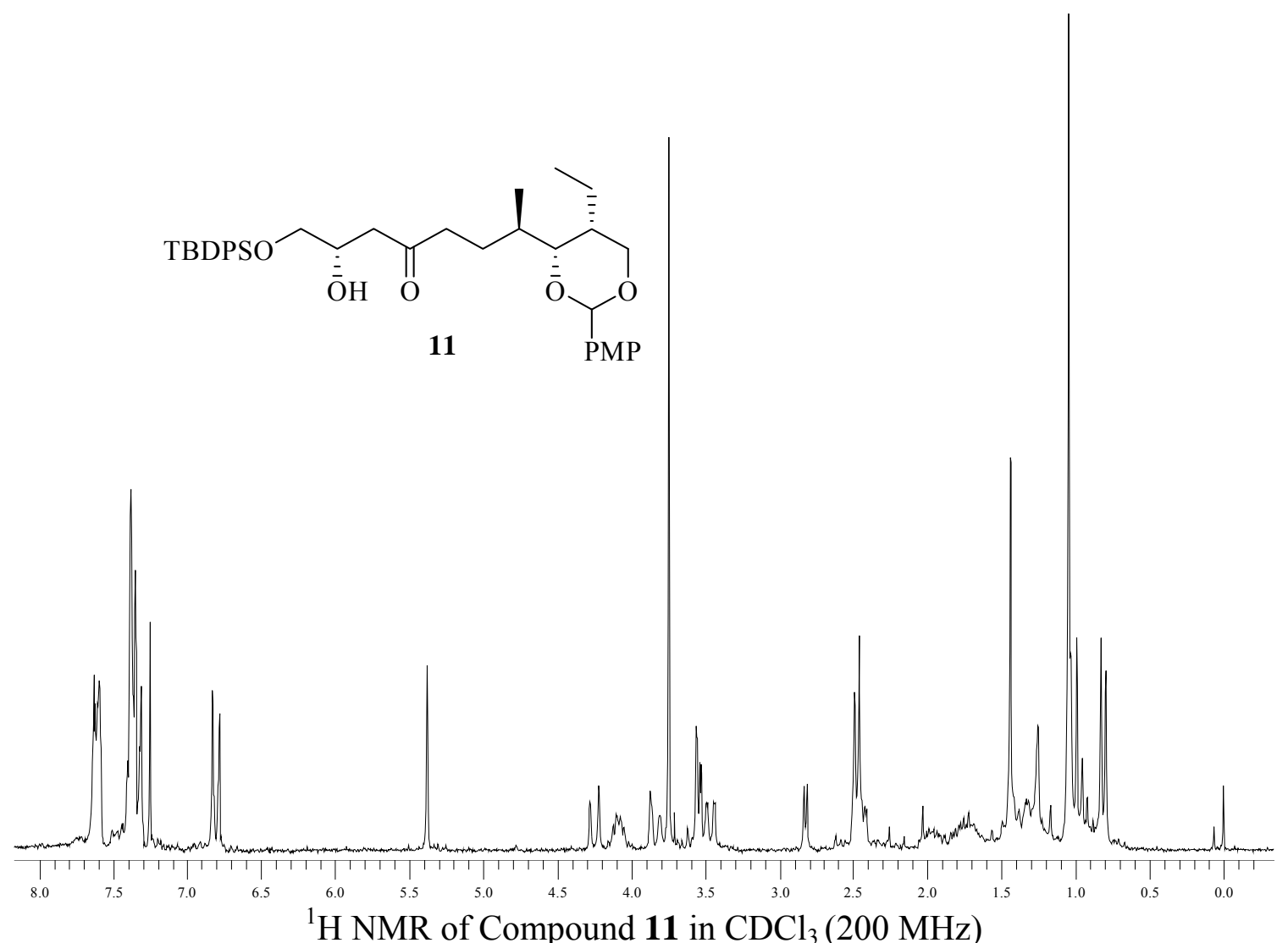

${ }^{1} \mathrm{H}$ NMR of Compound 11 in $\mathrm{CDCl}_{3}(200 \mathrm{MHz})$

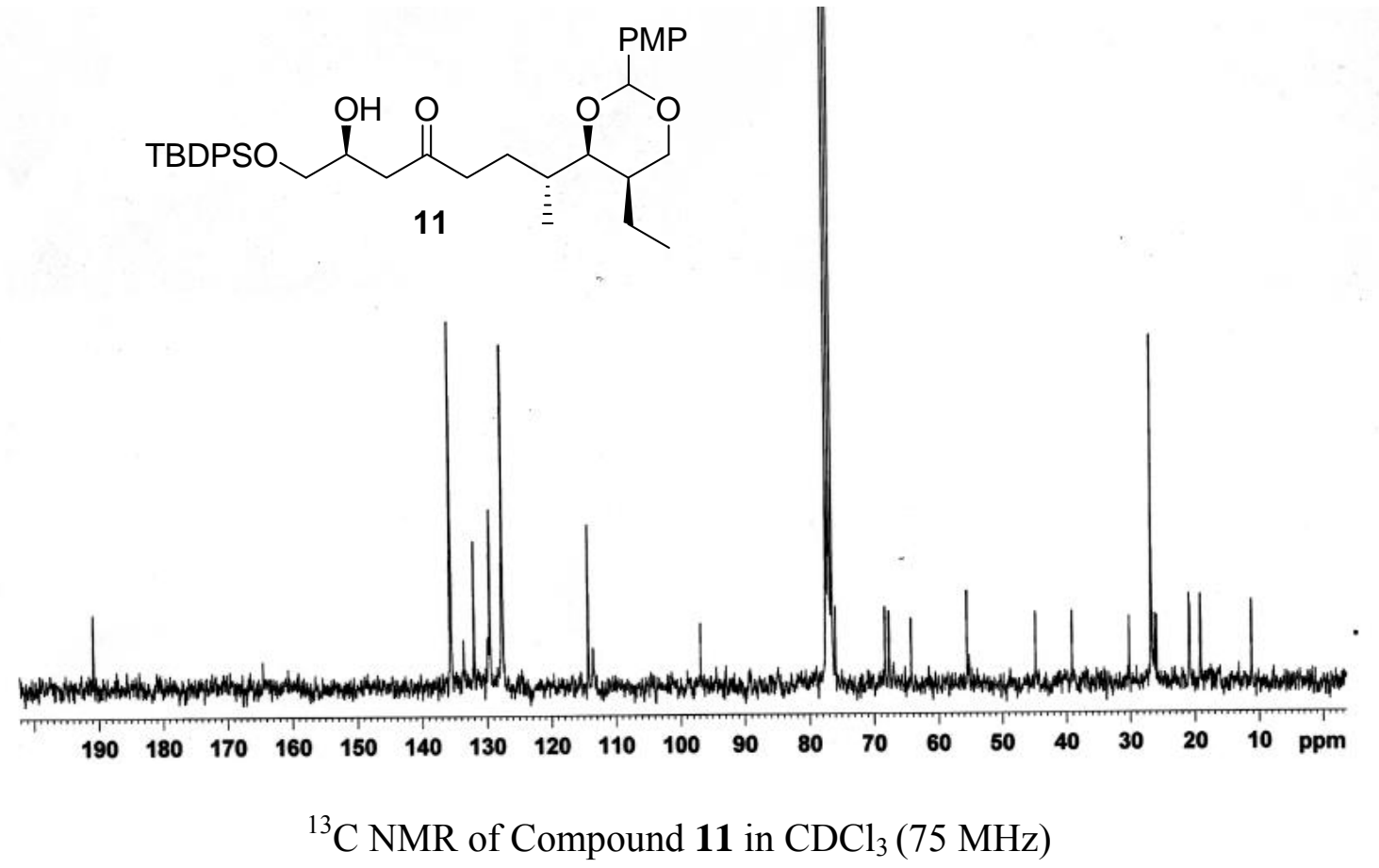



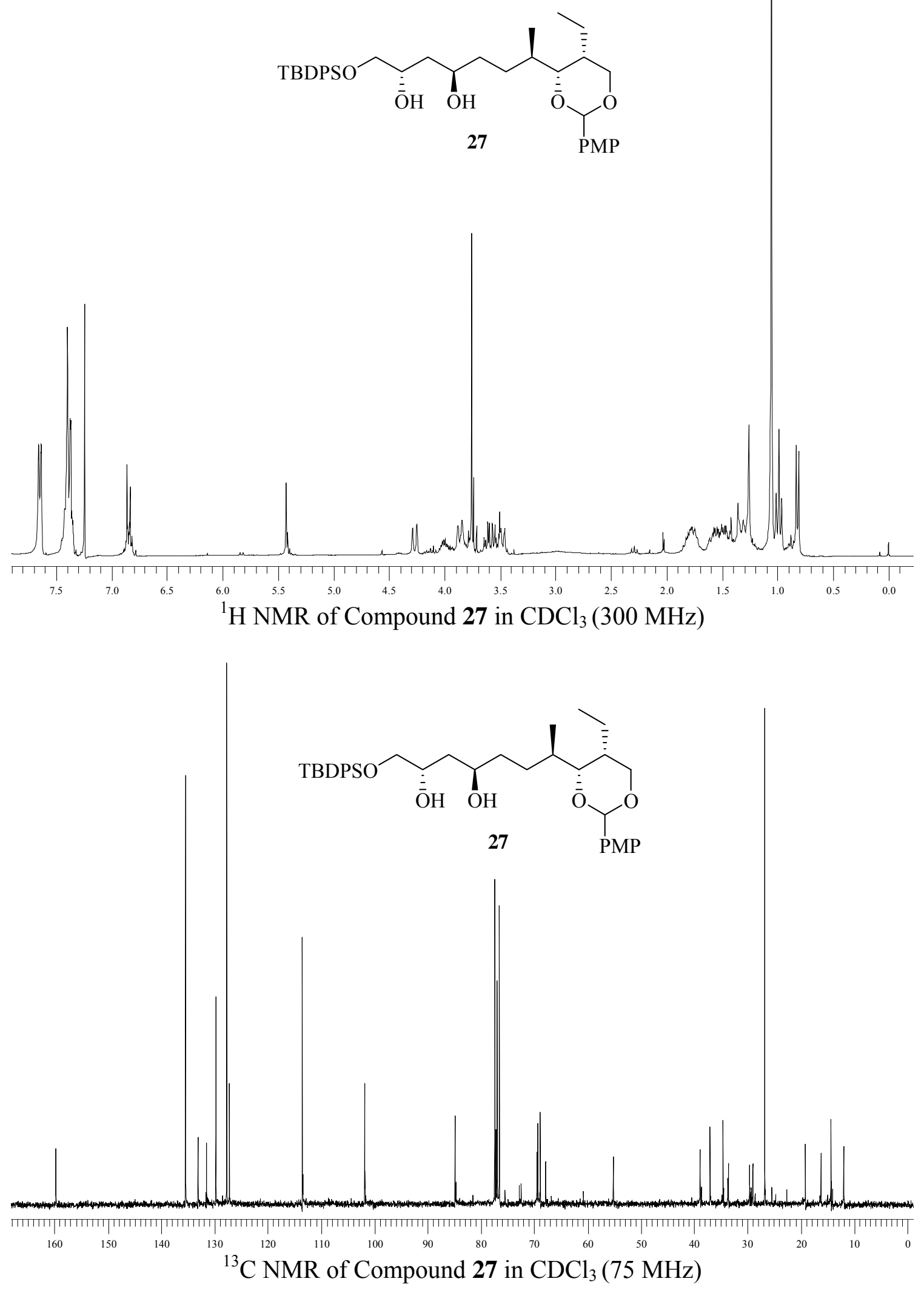

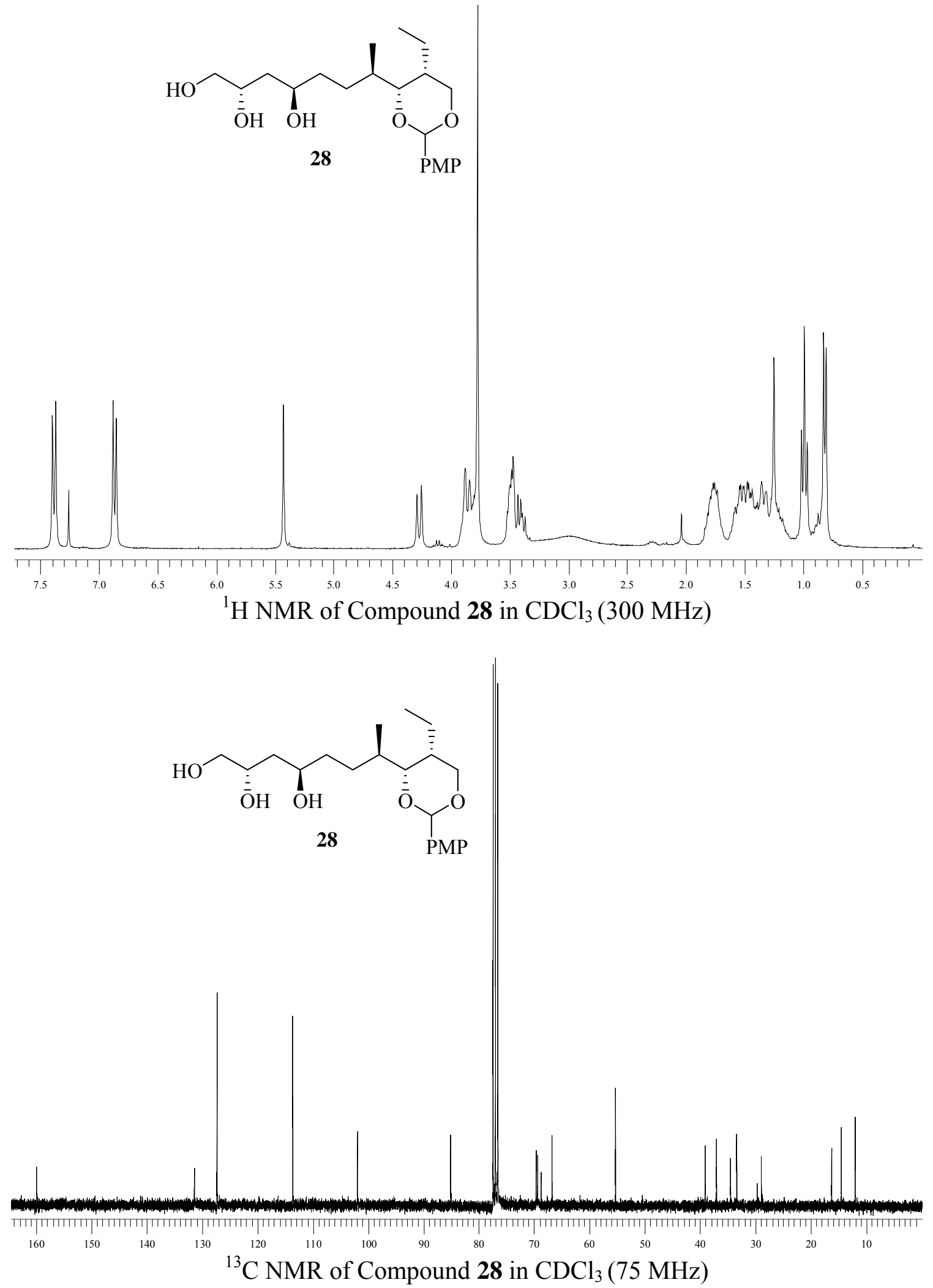

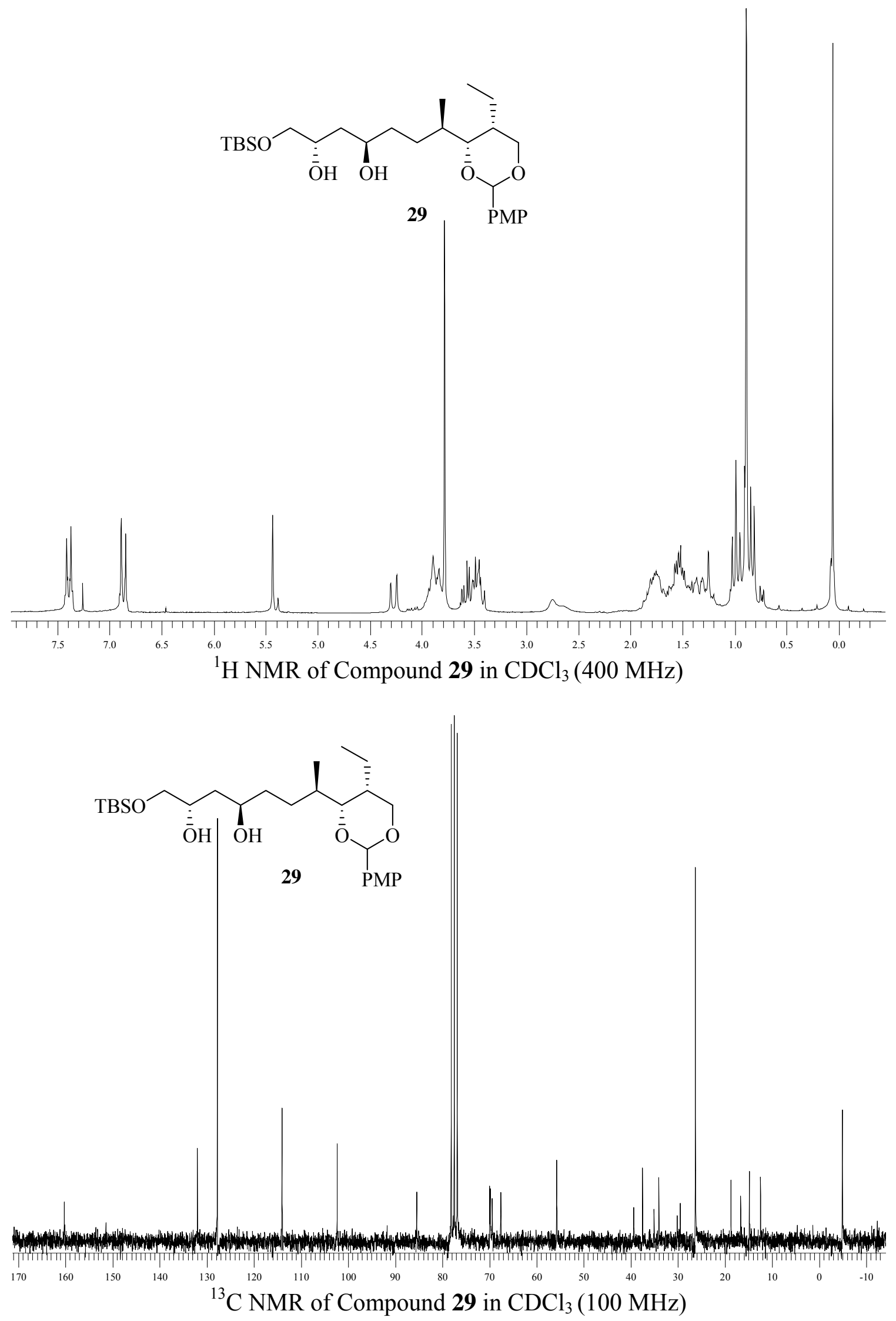

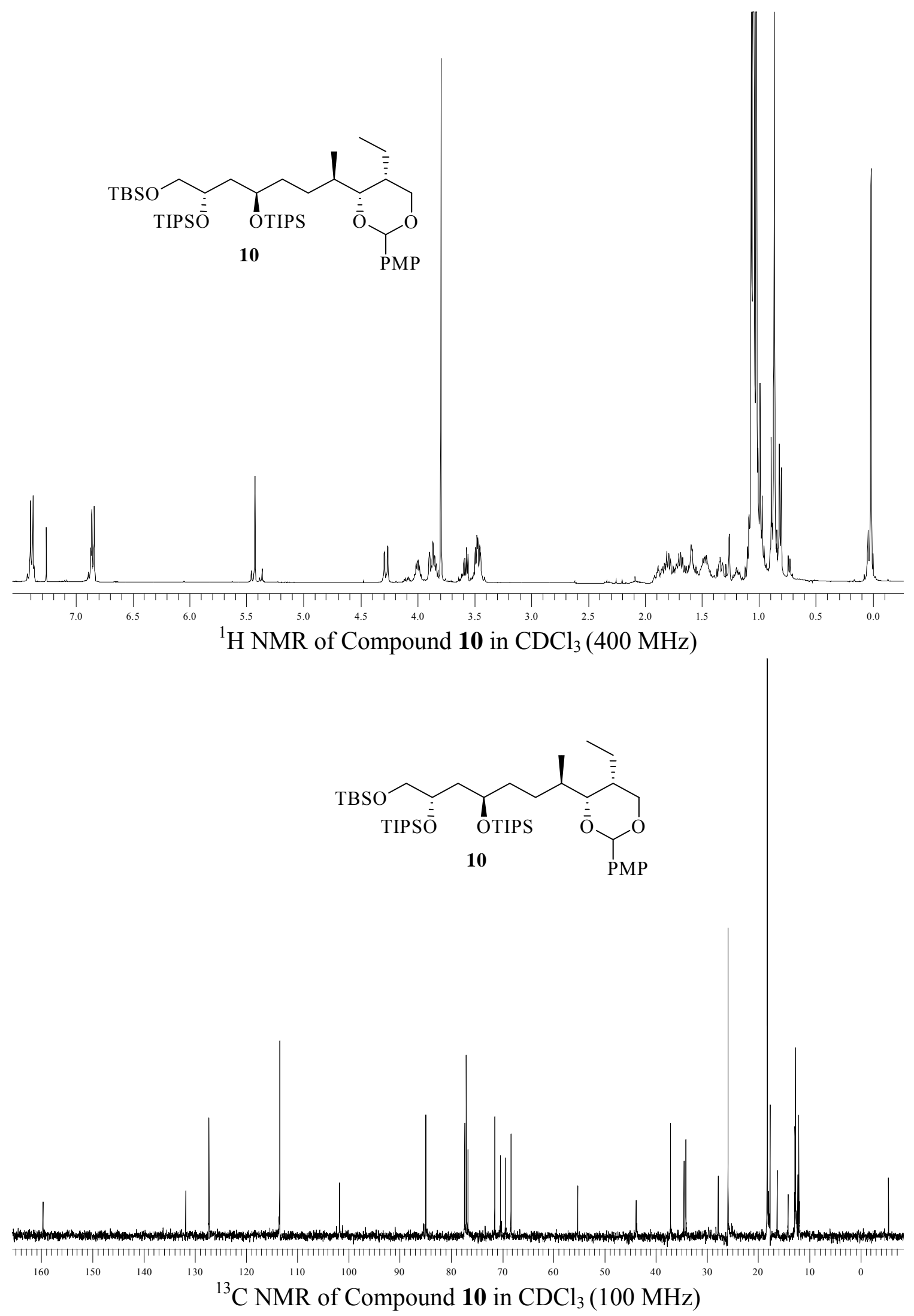

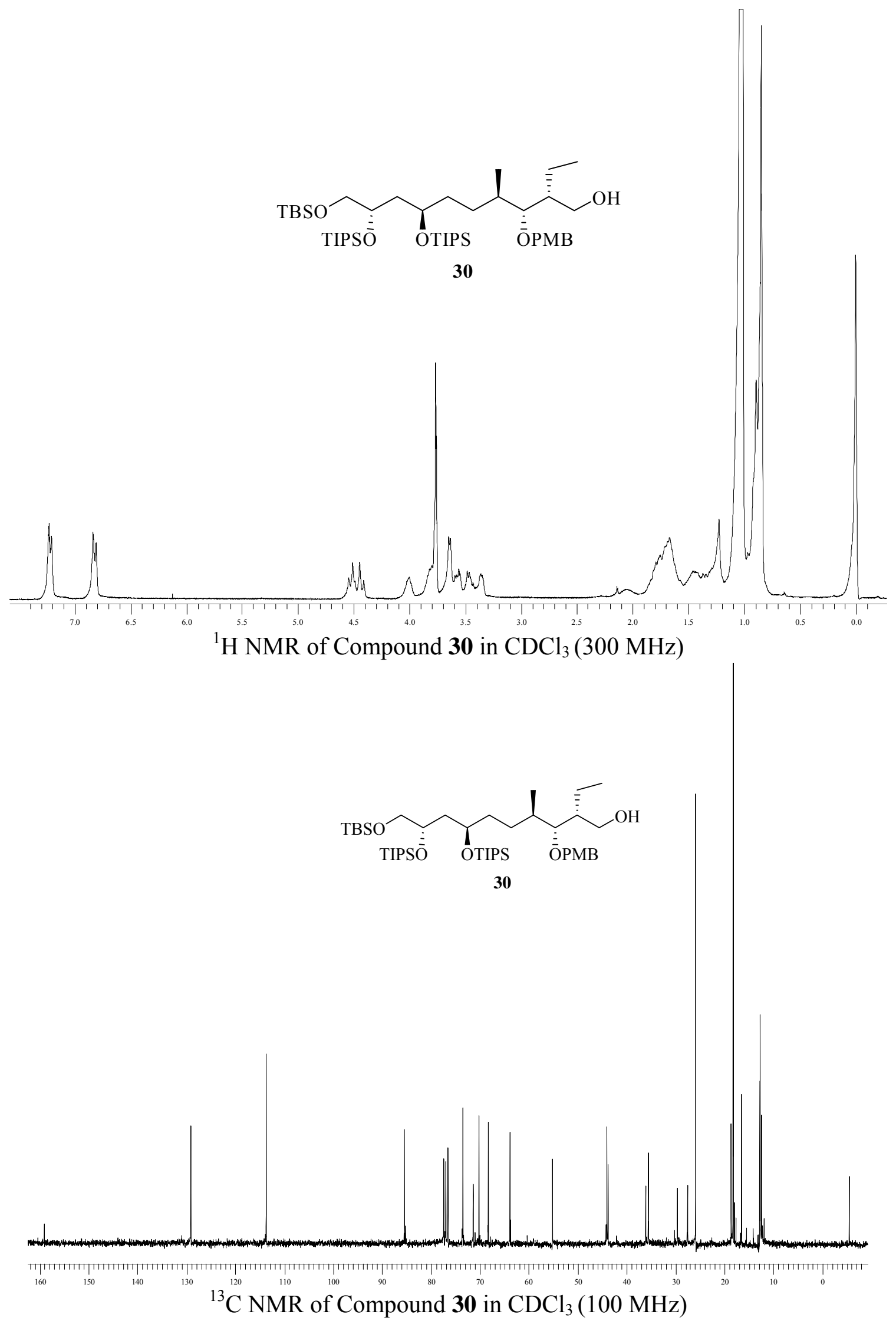

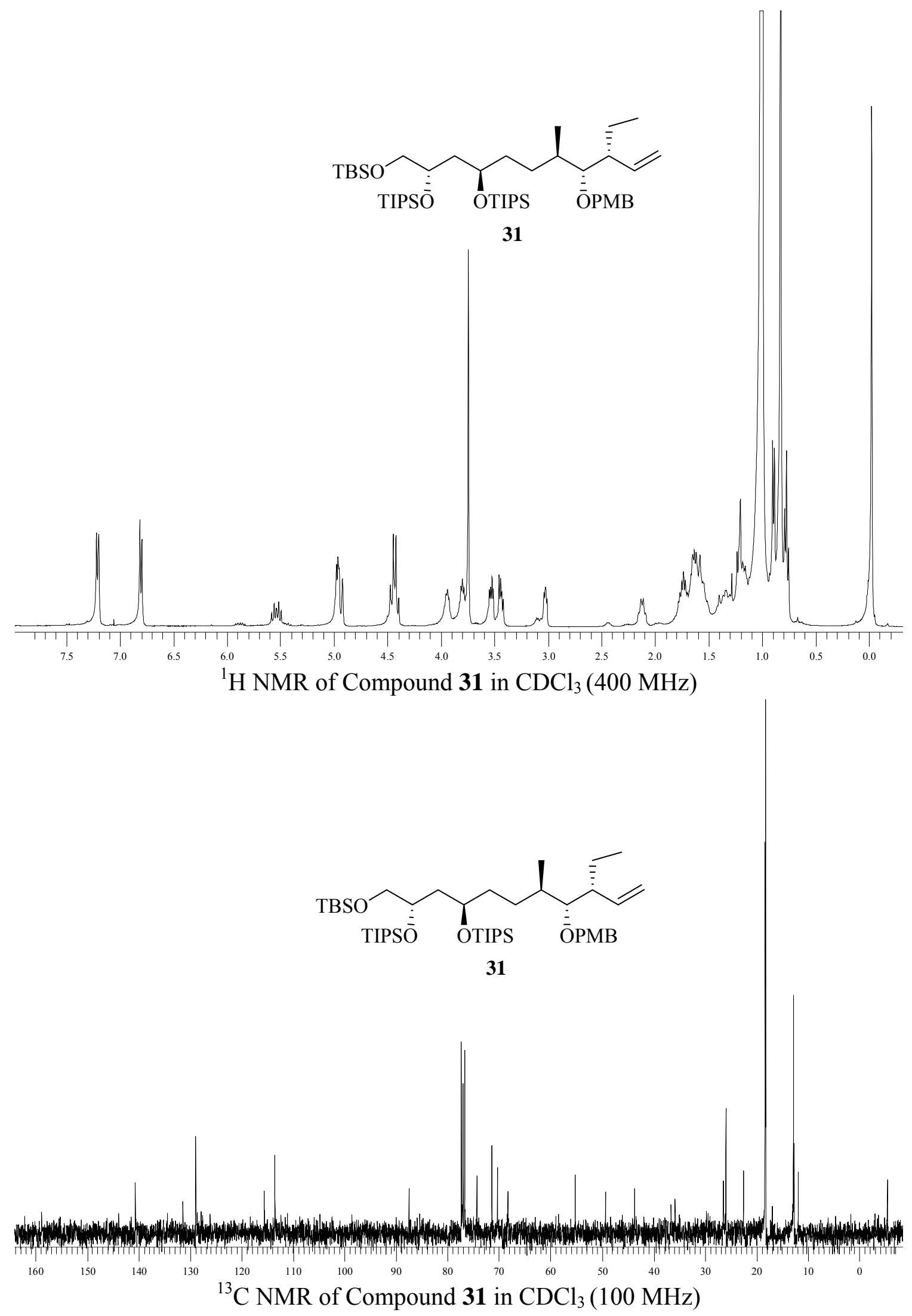

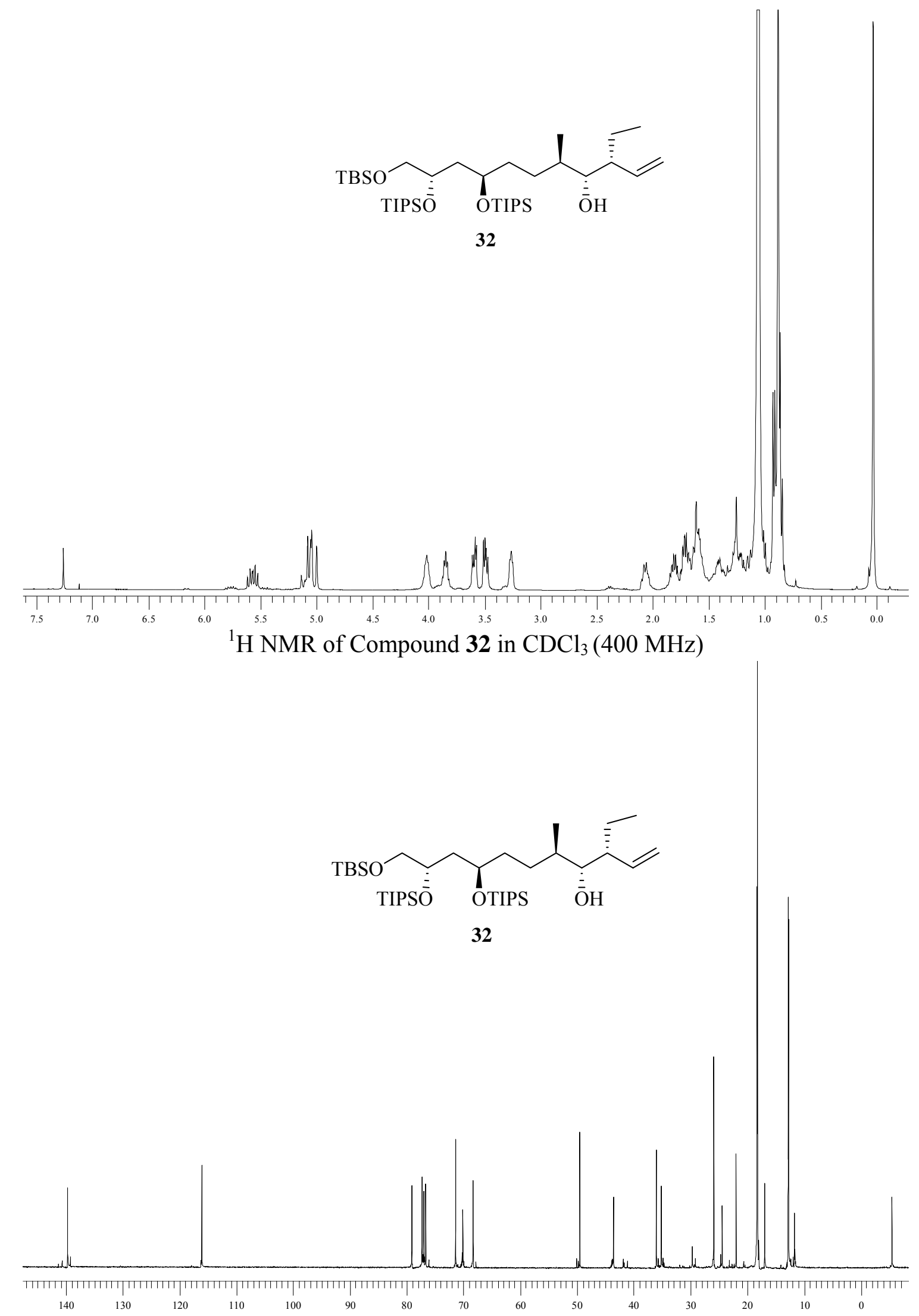
${ }^{13} \mathrm{C}$ NMR of Compound 32 in $\mathrm{CDCl}_{3}(100 \mathrm{MHz})$
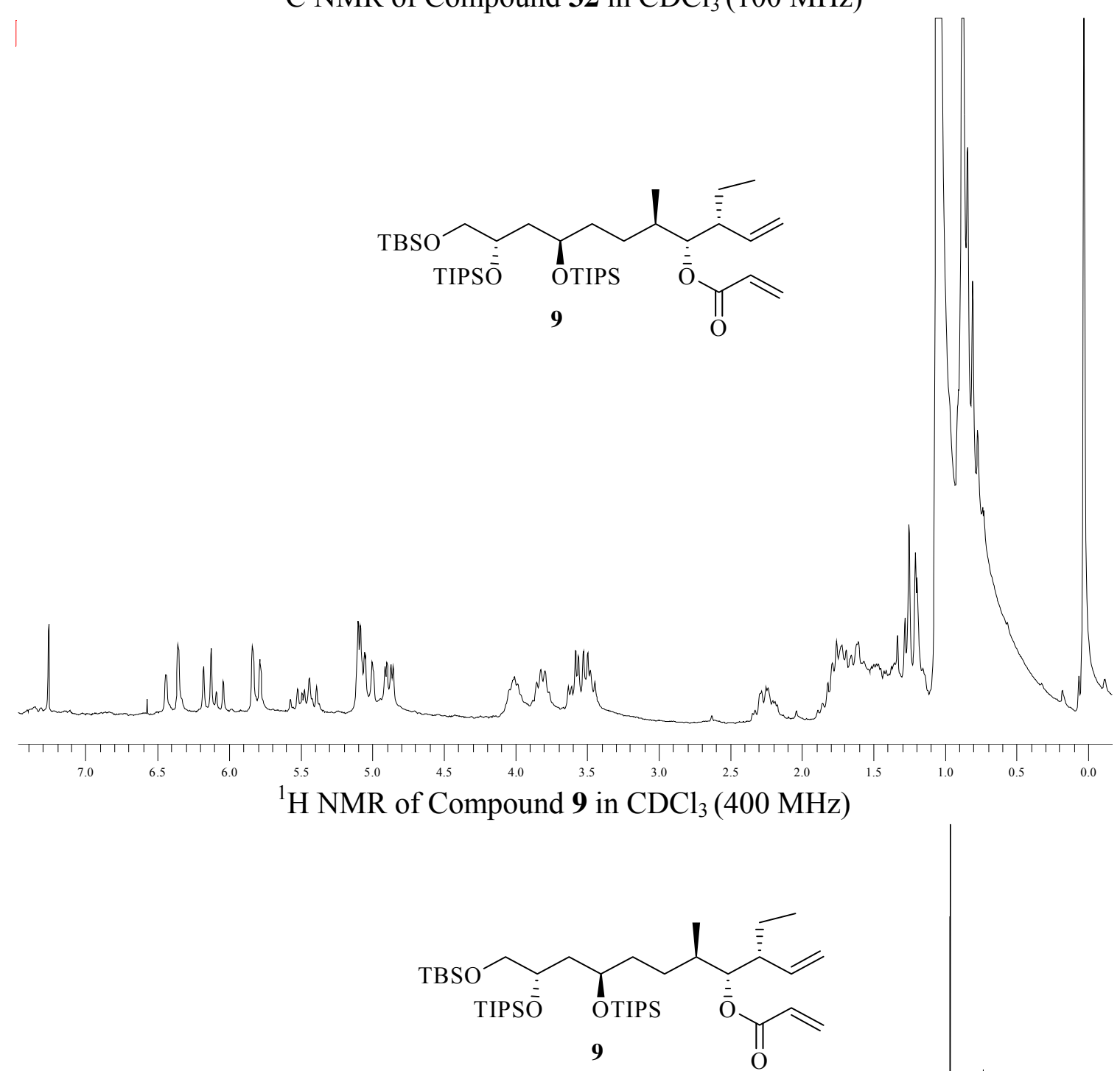

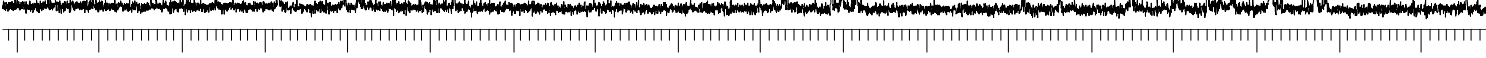
$\begin{array}{llll}170 & 160 & 150 & 140\end{array}$ 

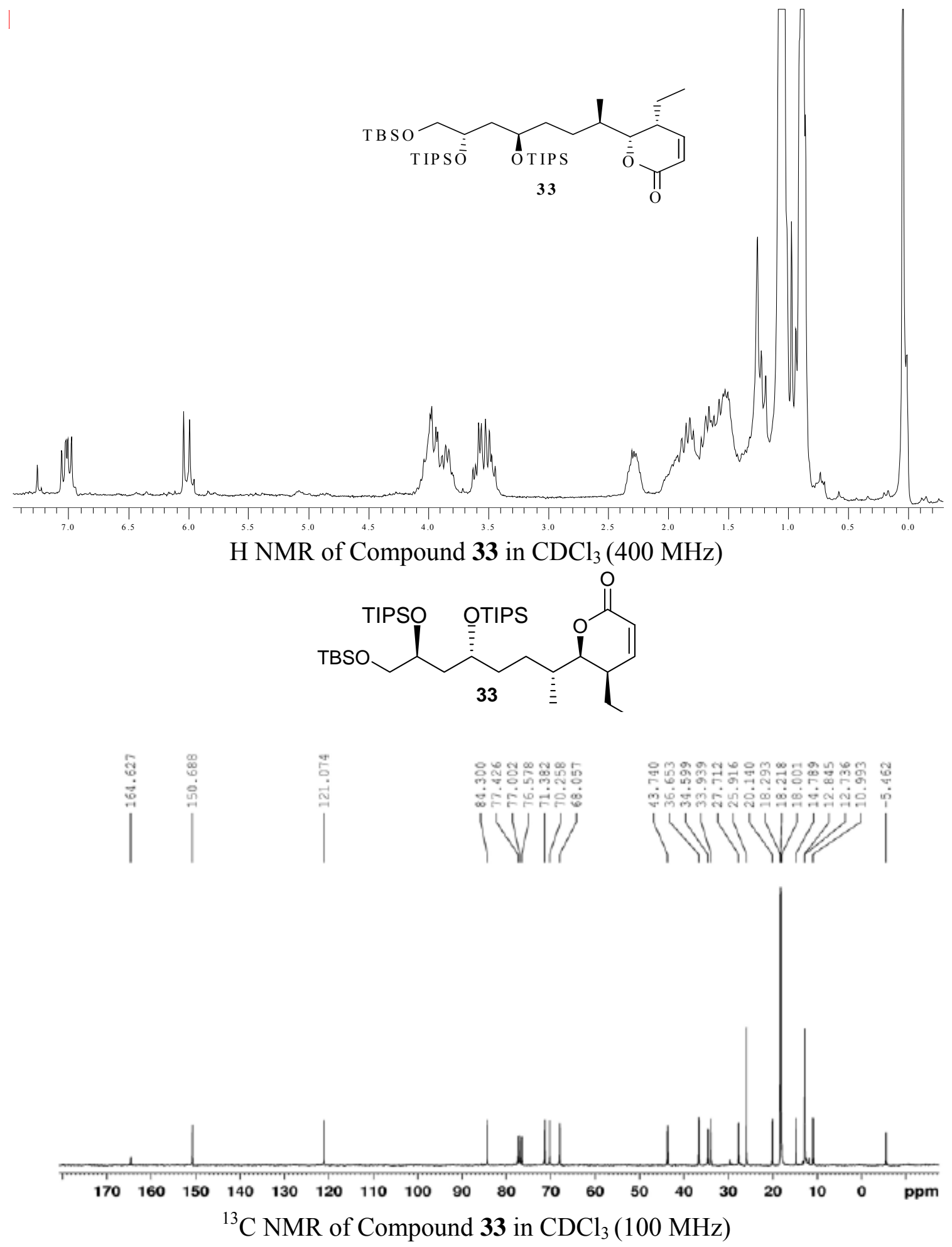

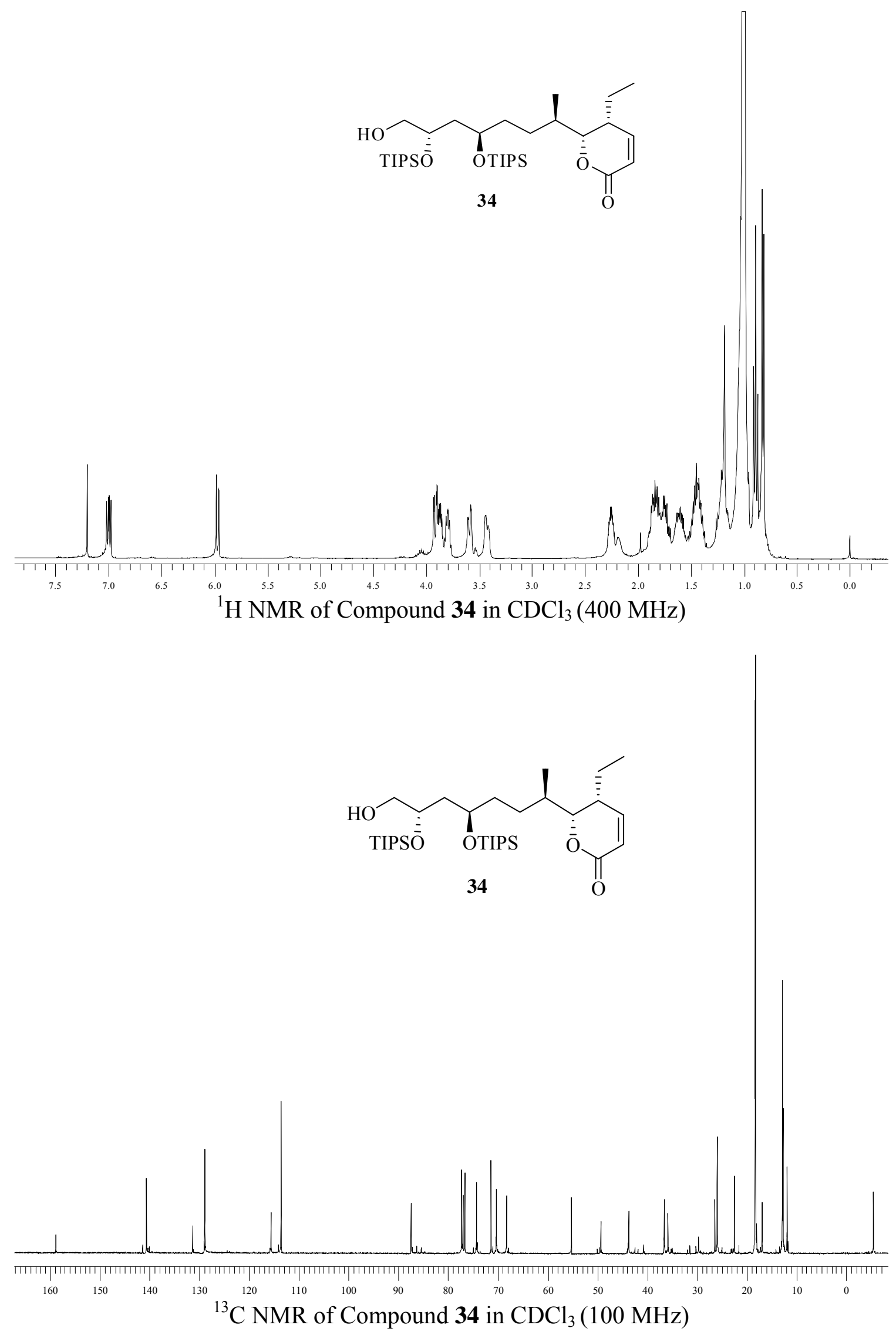

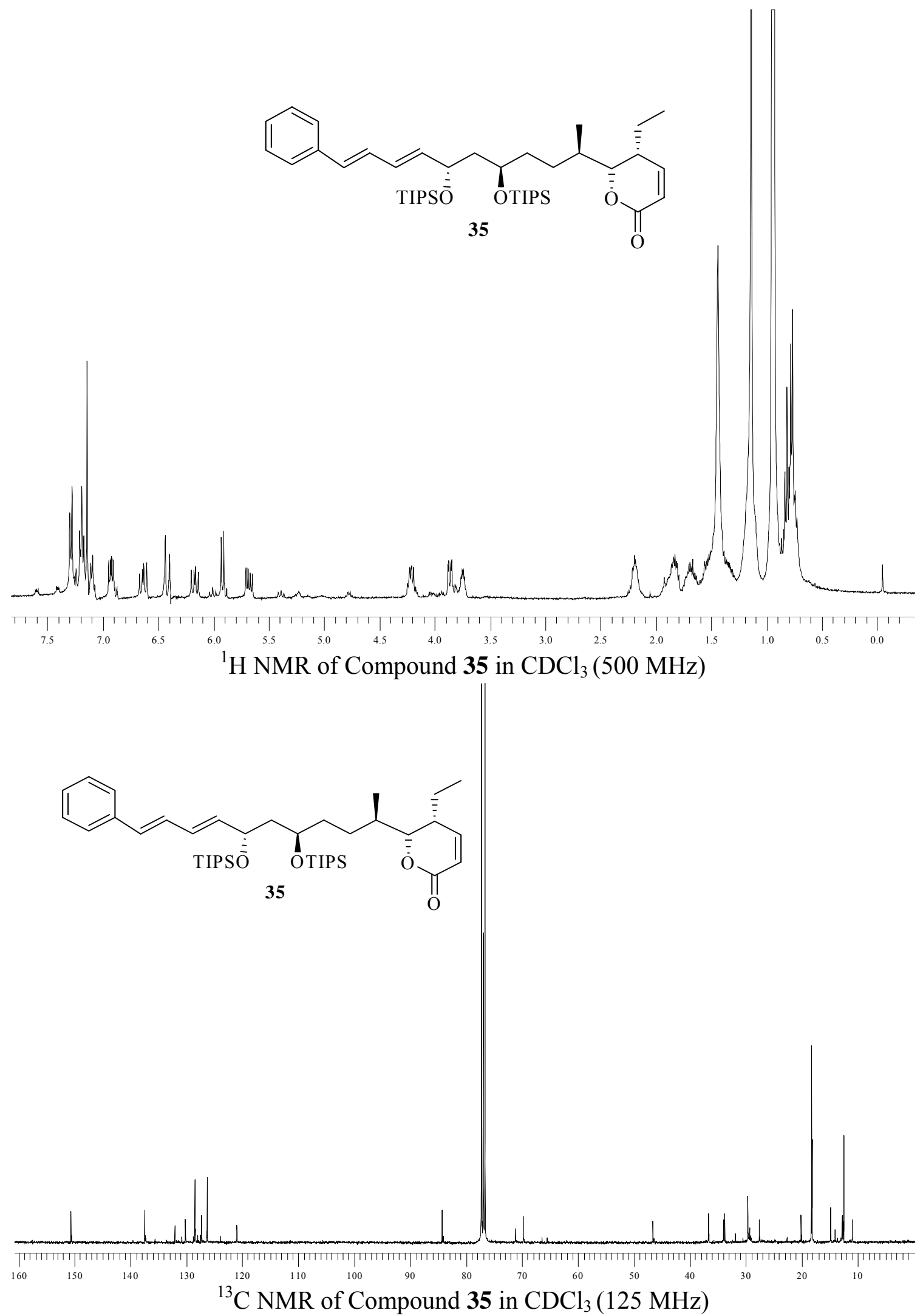


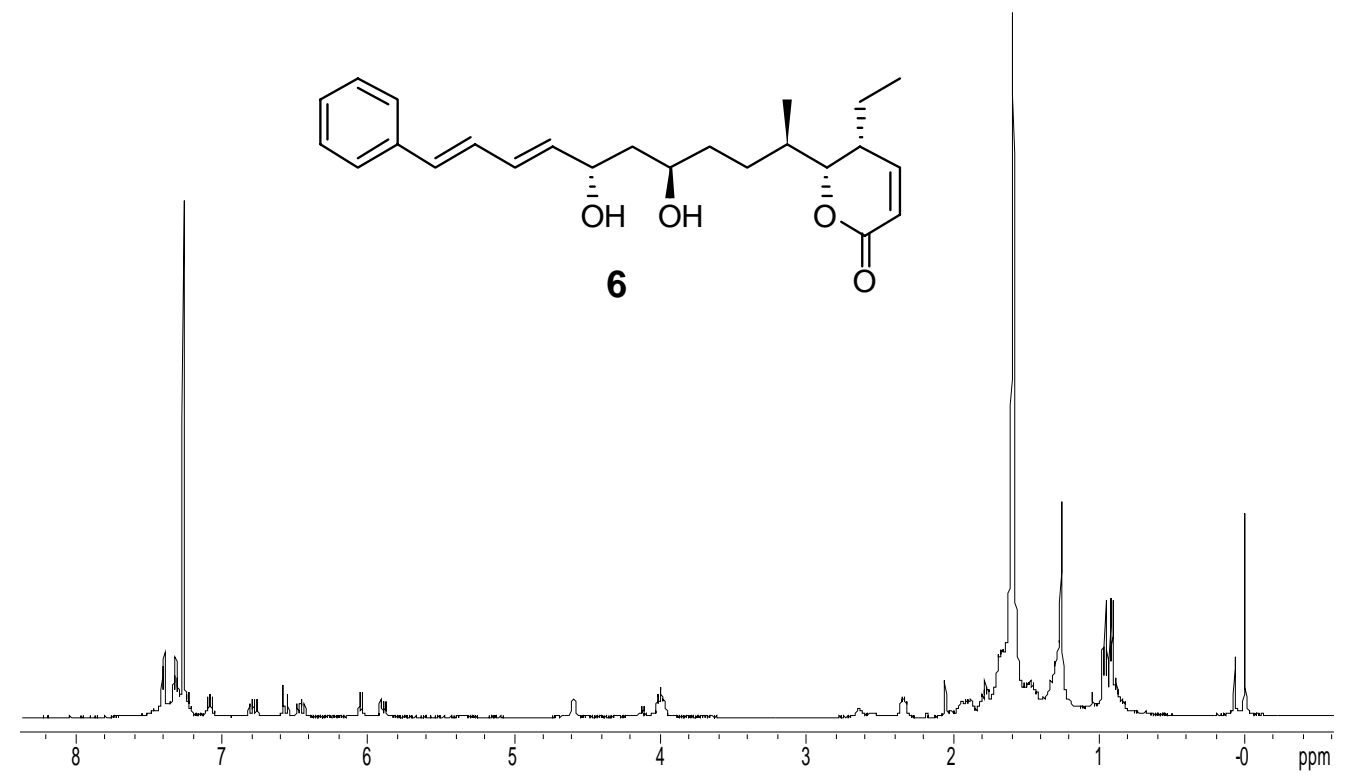

${ }^{1} \mathrm{H}$ NMR of Compound 6 in $\mathrm{CDCl}_{3}(500 \mathrm{MHz})$

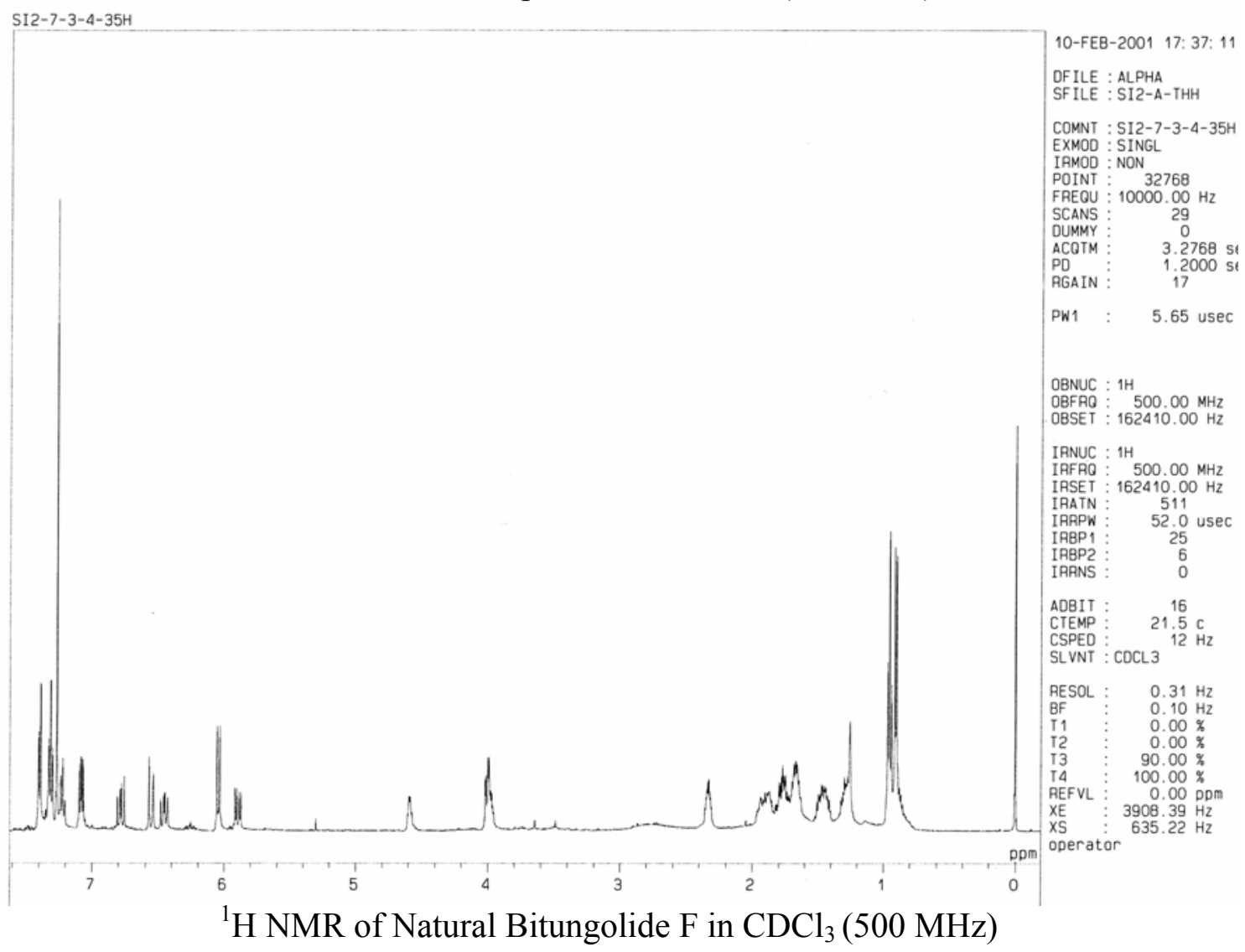


<smiles>CC[C@H]1C=CC(=O)O[C@H]1[C@@H](C)CC[C@@H](O)C[C@H](O)/C=C/C=C/c1ccccc1</smiles>
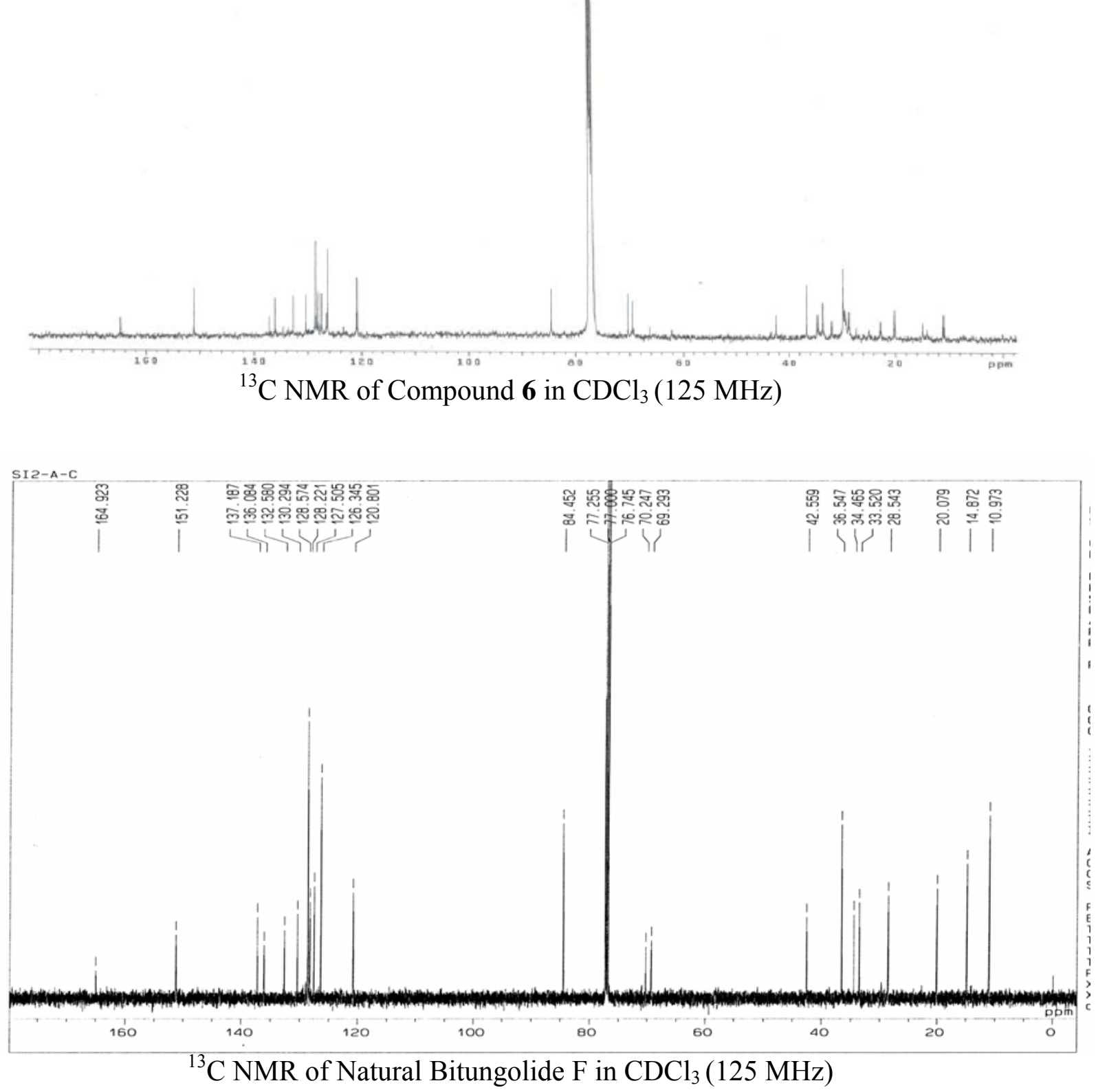